\title{
Electron Boltzmann kinetic equation averaged over fast electron bouncing and pitch-angle scattering for fast modeling of electron cyclotron resonance discharge
}

\author{
I. Kaganovich, ${ }^{1, *}$ M. Mišina, ${ }^{2, \dagger}$ S. V. Berezhnoi, ${ }^{3, \dagger}$ and R. Gijbels ${ }^{2, \S}$ \\ ${ }^{1}$ Department of Chemical Engineering, University of Houston, 4800 Calhoun Rd., Houston, Texas 77204-4792 \\ ${ }^{2}$ Department of Chemistry, University of Antwerp, Universiteitsplein 1, B-2610 Wilrijk, Belgium \\ ${ }^{3}$ Physical Technical Department, St. Petersburg State Technical University, 195251 St. Petersburg, Russia
}

(Received 7 June 1999)

\begin{abstract}
The electron distribution function (EDF) in an electron cyclotron resonance (ECR) discharge is far from Maxwellian. The self-consistent simulation of ECR discharges requires a calculation of the EDF on every magnetic line for various ion density profiles. The straightforward self-consistent simulation of ECR discharges using the Monte Carlo technique for the EDF calculation is very computer time expensive, since the electron and ion time scales are very different. An electron Boltzmann kinetic equation averaged over the fast electron bouncing and pitch-angle scattering was derived in order to develop an effective and operative tool for the fast modeling (FM) of low-pressure ECR discharges. An analytical solution for the EDF in a loss cone was derived. To check the validity of the FM, one-dimensional (in coordinate) and two-dimensional (in velocity) Monte Carlo simulation codes were developed. The validity of the fast modeling method is proved by comparison with the Monte Carlo simulations. The complete system of equations for FM is presented and ready for use in a comprehensive study of ECR discharges. The variations of plasma density and of wall and sheath potentials are analyzed by solving a self-consistent set of equations for the EDF.
\end{abstract}

PACS number(s): 52.50.Gj, 52.65.Ff, 52.65.Pp

\section{INTRODUCTION}

In recent years, low-pressure, high-density plasma sources have attracted considerable interest for application in material processing, e.g., etching, deposition, etc. [1]. One of the possible mechanisms for plasma generation in these sources is electron cyclotron resonance (ECR) heating. Typically, the operating gas pressures in ECR reactors range from $10^{-5}$ to $10^{-2}$ Torr. Correspondingly, the electron mean free path can be larger than or comparable to the discharge dimensions (typically several tens of centimeters). Under these conditions, the electron distribution function (EDF) is far from Maxwellian, and can be enriched by slow electrons [2]; moreover, the EDF can have a pronounced high-energy tail [3]. A non-Maxwellian EDF is associated with a number of nonlinear phenomena of the discharge self-organization. The high-energy electrons determine the dissociation and ionization rates. The slow electrons are responsible for the formation of the ambipolar potential in the plasma bulk. It was demonstrated [4] that the formation of a two-temperature EDF can be accompanied by an explosive generation of the bulk plasma density. The distribution of electron temperature in the direction transverse to the magnetic field effectively influences the plasma profile [5]. Since the EDF form is of primary importance in low-pressure discharges, it is necessary to solve the kinetic equation for the electrons even for a qualitative description of such discharges. For these reasons, the EDF in ECR discharges should be fully addressed.

\footnotetext{
*Electronic address: ikaganov@ jetson.uh.edu

†Electronic address: misina@fzu.cz

*Electronic address: berezhnoj@phtf.stu.neva.ru

${ }^{\S}$ Electronic address: gijbels@uia.ua.ac.be
}

Mechanisms of the formation of non-Maxwell EDF's were investigated in detail experimentally and numerically, mainly in capacitively and inductively coupled plasmas $[6,7]$, and in dc discharges [8].

A natural approach to the modeling of discharges at low pressures is the use of the particle-in-cell (PIC) computer simulation along with the Monte Carlo (MC) technique [9]. However, the enormous range of the spatial and temporal scales precludes a straightforward application of the PIC and MC methods to the self-consistent modeling of discharges. The highest characteristic frequency is the electron plasma frequency $\omega_{\mathrm{ep}} \sim 10^{10} \mathrm{~s}^{-1}$, and the electron cyclotron frequency $\omega_{\mathrm{ec}} \sim 10^{10} \mathrm{~s}^{-1}$, whereas the ion residence time in the discharge is about $10^{-4} \mathrm{~s}$. The characteristic spatial scales in the Poisson equation, i.e., the Debye radius $r_{d}$ and the sheath thickness, are about $1 \mathrm{~mm}$, whereas the discharge dimensions are $10-30 \mathrm{~cm}$. To eliminate small spatial and temporal time scales, one has to use the quasineutrality condition instead of the Poisson equation. The PIC with MC treatment of collisions and the quasineutrality equation for the electric field were combined for the self-consistent modeling of an ECR discharge in Refs. [10,11]. Electrons were treated by the guiding center approximation. This approximation excludes the electron gyro time scale from the dynamics. The fastest temporal scale left in this scheme is the electron bounce time $t_{b} \sim L / \mathrm{v}_{e}$, which is about 100 times shorter than the ion lifetime $\gtrsim L / \mathrm{v}_{i}$.

An averaging procedure is needed to avoid resolving the time scale of the electron bouncing. A natural approach is to model the discharge using a space-time-averaged kinetic equation. This approach allows one to perform simulations for a wide range of parameters and even simultaneously with experiments. Moreover, one can analyze in detail complicated interactions of different physical processes, which is not possible otherwise [4]. 
The kinetic analysis of the self-consistent motion of electrons and ions in capacitively coupled plasma (CCP) discharges has been reported earlier [12]. It is based on the theory developed in Refs. $[13,14]$. Fast modeling (FM) for the case of an inductively coupled plasma (ICP) was presented in many papers (see, e.g., Refs. $[15,16])$. The success in the modeling of CCP and ICP has encouraged us to develop a similar method for ECR modeling.

The kinetic equations averaged over electron bouncing were derived for an EDF study in ECR discharges in several papers $[17,18,19]$. In all these papers pitch-angle scattering due to collisions with neutrals was neglected. The results of MC modeling [20] and theoretical estimates [14] show that the largest part of the electrons should be trapped in an electrostatic potential well. As a result of the long residence time in the potential trap, exceeding the electron-atom collision time, the EDF is close to an isotropic function even though the electron mean free path can be large compared to the discharge dimensions.

For the calculation of the EDF in ECR discharges, in this paper we present an electron Boltzmann kinetic equation (zero dimensional in space, and one dimensional in energy) averaged over fast electron bouncing and over elastic collisions, which is similar to what was done in Refs. [12-16]. An analytical solution for the EDF in the loss cone is derived. The validity of the FM method is proved by comparison with Monte-Carlo simulations (one dimensional in space, and two dimensional in velocity). Examples of selfconsistent simulations are shown in Sec. IV.

\section{DESCRIPTION OF THE MODEL}

The developed theory is applicable to a wide range of conditions. However, to be more specific, we consider a conventional ECR source with a microwave frequency of 2.45 $\mathrm{GHz}$. The electron resonance occurs at a magnetic field equal to $875 \mathrm{G}$. We assume that the magnetic field is monotonically decreasing (known as a magnetic beach), so that there is only one point of resonance. The pressure is taken in the range of $0.1-10 \mathrm{~m}$ Torr. The background gas is argon. An axisymmetric chamber of a width $L=20 \mathrm{~cm}$ was considered. All electron drifts are azimuthal because of the symmetry. The electron gyro radius is extremely small $(<0.1 \mathrm{~mm})$. Thus electrons are tied to a fixed flux tube. Furthermore, we may assume that the electron motion is one dimensional, since the azimuthal motion can be ignored in the cylindrical geometry.

The averaged electron energy is determined from a balance of the ionization and particle losses, and is typically a few eV. This implies that the ambipolar potential should also be of the order of several V. The plasma density varies from $10^{9}$ to $10^{13} \mathrm{~cm}^{-3}$ in ECR discharges. The amplitude of the electric field in the wave is a few eV/cm. The main interaction of electrons with a wave in the resonance region is defined by $\omega_{e c} \equiv[e B(x) / m c]=\omega$, where $\omega$ is the wave frequency, $B$ is the magnetic field, $m$ is the electron mass, $e$ is the elementary charge, and $c$ is the velocity of the light in a vacuum. Electrons effectively gain or lose energy from or to the wave, until the phase of the gyration and the wave phase depart $\int([e B(x) / m c]-\omega) d t \sim \pi[1]$. This implies that the electrons with parallel velocity $\mathbf{V}_{\|}$interact with a wave effi- ciently during a time $t_{r}=\sqrt{2 \pi} / \sqrt{\mathbf{V}_{\|}\left|d \omega_{\mathrm{ec}} / d x\right|}$ and within a distance $d x_{r}=\sqrt{2 \pi \mathrm{v}_{\|}} / \sqrt{\left|d \omega_{\mathrm{ec}} / d x\right|}$. Under the conditions discussed, $d x_{r} \sim 1 \mathrm{~cm} \ll L$ and the resonance region can be considered as narrow. The change in energy after passing the resonance zone (a "kick") is $\Delta \varepsilon_{\perp} \equiv m \mathbf{v}_{\perp} \Delta \mathbf{v}_{\perp} \sim$ $\left.\pm\left(e E_{\perp} \mathbf{V}_{\perp} d x_{r}\right) / \mathbf{V}_{\|}\right)$, where $\mathbf{V}_{\perp}$ is the perpendicular velocity and $E$ is the amplitude of the wave electric field, and is of the order of $1 \mathrm{eV} / \mathrm{cm}$. Since the kick in energy is much smaller than the energy itself, the series of subsequent kicks can be described as a diffusion in the energy space.

\section{ELECTRON-WAVE INTERACTION IN THE RESONANCE POINT}

In this section we shall briefly summarize the main results of the electron interaction with a wave in the resonant point. The nonrelativistic equation for the complex perpendicular velocity $\mathrm{v}_{\perp}=\mathrm{v}_{z}+i \mathrm{v}_{y}$ reads

$$
\frac{d}{d t} \mathrm{v}_{\perp}+i \omega_{e c} \mathrm{v}_{\perp}=-\frac{e}{m} E_{\perp} e^{-i \varphi_{w}(t)},
$$

where $E_{\perp}$ is the amplitude of the wave electric field, $\varphi_{w}$ is the phase of the wave, and $d \varphi_{w} / d t=\omega$. The integration of Eq. (1) from the time- $T$ to the time $T$ yields [21]

$$
\mathrm{v}_{\perp}(T)=\left[V_{p}(T)+\mathrm{v}_{\perp}(-T)\right] e^{i \varphi_{g}(T)},
$$

where $\varphi_{g}(T)=\int_{-T}^{T}[e B(x) / m c] d t+\varphi_{0}$ is the phase of the electron gyration, and

$$
V_{p}(T)=\frac{e}{m} E_{\perp} \int_{-T}^{T} e^{i\left(\varphi\left(t^{\prime}\right)-\varphi_{w}\left(t^{\prime}\right)\right)} d t^{\prime}
$$

is the change in velocity due to the interaction with the electric field of a wave. The maximum contribution in integral (2b) is at the point of resonance $t=0, d \varphi_{w} / d t=d \varphi_{g} / d t$. The absolute velocity of $\left|V_{p}\right|$ nearly does not vary when the electrons pass resonance for times longer than the interaction time $t_{r}$. This means that the time $T$ can be extended to infinity;

$$
V_{p}=\frac{e}{m} E_{\perp} \int_{-\infty}^{\infty} e^{i\left(\varphi_{g}\left(t^{\prime}\right)-\varphi_{w}\left(t^{\prime}\right)\right)} d t^{\prime} \approx \frac{e}{m} E_{\perp} t_{r}
$$

where

$$
\begin{aligned}
t_{r} & =\int_{-\infty}^{\infty} e^{i\left(\varphi_{g}\left(t^{\prime}\right)-\varphi_{w}\left(t^{\prime}\right)\right)} d t^{\prime} \approx e^{\theta i} \int_{-\infty}^{\infty} e^{i \varphi_{g}^{\prime \prime} t^{2} / 2} d t \\
& =\sqrt{\left(\frac{2 \pi}{\varphi_{g}^{\prime \prime}}\right)} e^{i(\theta \mp \pi / 4)},
\end{aligned}
$$

$\theta=\varphi_{g}(0)-\varphi_{w}(0)$ is the difference of the electron gyro rotation phase and the wave phase at the moment of resonance $\omega_{e c}=\omega, \varphi_{g}^{\prime \prime}=\left(d \omega_{c} / d x\right) \mathbf{V}_{\|}$; the minus sign corresponds to a positive $\varphi_{g}^{\prime \prime}$, and the plus sign to a negative $\varphi_{g}^{\prime \prime}$.

From Eq. (2) one can find the energy change in the resonance in forms [21]

$$
\varepsilon_{\perp(n+1)}=\varepsilon_{\perp n}+\varepsilon_{p}+2 \sqrt{\varepsilon_{\perp n} \varepsilon_{p}} \cos \left(\theta_{n+1} \mp \pi / 4\right),
$$




$$
\theta_{n+1}=\theta_{n}+\int_{T_{n}}^{T_{n+1}}\left(\frac{e B(x)}{m c}-\omega\right) d t
$$

where $\varepsilon_{p} \equiv(m / 2)\left|V_{p}\right|^{2}$, and $n$ denotes subsequent interactions at moments $T_{n}$. The integral $\int_{T_{n}}^{T_{n+1}}([e B(x) / m c]$ $-\omega) d t$ denotes the number $(2 \pi N)$ of rotations with angle velocity $\left(\omega_{e c}-\omega\right)$ during one bounce of electron along the discharge. If the magnetic field variation along the discharge is not too small, $\omega_{\mathrm{ec}}-\omega \sim \omega$, we can estimate $N \sim t_{b} \omega / 2 \pi$, where $t_{b}$ is the bounce period. Under our conditions, $N$ $\sim 1000$. Analyzing mapping (3), one can find a condition for an instability of the trajectory $\left(\varepsilon_{\perp n}, \theta_{n}\right)$. The trajectory instability leads to a stochastic motion. A rough criterion consists in the following condition [22]: The motion is stochastic if the kick in energy in the resonance results in a variation of the next phase by more than $\left|\delta \theta_{n+1}\right|>\pi$ or $|\delta N|>1 / 2$. The electrons move along a magnetic line in an effective potential $-e \Phi(x)+\mu B(x)$, where $\Phi(x)$ is the electrostatic potential and $\mu=m \mathrm{v}_{\perp}^{2} / 2 B$ is the first adiabatic invariant, with a constant total energy $\varepsilon_{\|}=0.5 m \mathrm{v}_{\|}^{2}-e \Phi(x)+\mu B(x)$. The values of $\varepsilon_{\|}, \mu$ characterize the trajectory and, correspondingly, the number of rotations. A variation in the perpendicular energy changes values of $\varepsilon_{\|}, \mu$, and $N$, respectively. So the criterion for stochastic motion can be written in the form

$$
\left|\frac{\partial N\left(\varepsilon_{\|}, \mu\right)}{\partial \varepsilon_{\perp}} \Delta \varepsilon_{\perp}\right|>1 / 2,
$$

where $\Delta \varepsilon_{\perp}=\varepsilon_{p}+2 \sqrt{\varepsilon_{\perp n} \varepsilon_{p}} \cos \left(\vartheta_{n+1} \mp \pi / 4\right)$. Since $N$ is very large $(\sim 1000)$, even a small dependence of $N$ on $\varepsilon_{\|}, \mu$ will result in a stochastic motion. Thus in what follows we shall assume that criterion (4) is fulfilled, and consider a value $\theta_{n+1}$ as independent from the previous one $\theta_{n}$. In other words, the phases of subsequent interactions are assumed to be random. The more complicated case, when criterion (4) is not valid, was considered in a number of papers; see, e.g., Ref. [22]. In this case stochastization can occur due to the pitch-angle scattering [23,24].

Under the assumption of random phases of subsequent interactions, the integral describing electron interaction with the electric field can be modeled by a diffusion in the magnetic moment. The exact derivation can be found elsewhere, see, e.g., Refs. [18,19,22,23]. The most efficient method of derivation was proposed in Ref. [25]. Considering times longer than $t_{r}$ and distances larger than $d x_{r}$, the electronwave interaction integral can be written in the form [25]

$$
\begin{gathered}
S_{t}^{\mathrm{ECR}}(f)=\mathrm{v}_{\|} \delta\left(x-x_{r}\right) \frac{\partial}{\partial \mu} D_{\mu} \frac{\partial f}{\partial \mu}, \\
D_{\mu}=0.5\left\langle(\Delta \mu)^{2}\right\rangle=\frac{\mu e c E_{\perp}^{2}}{B} \frac{\pi}{\mathbf{v}_{\|}\left|\frac{d B}{d x}\right|},
\end{gathered}
$$

where angular brackets denote averaging over phases $\theta_{n}$. It is presumed that the kick in the perpendicular energy is small compared with the energy.

\section{AVERAGING PROCEDURE OVER FAST ELECTRON BOUNCING AND PITCH-ANGLE SCATTERING FOR TRAPPED ELECTRONS}

We start from the stationary electron Boltzmann kinetic equation in the drift approximation for the distribution function $f\left(\mathrm{v}_{\|}, \mu, x\right)$ :

$$
\mathbf{v}_{\|} \frac{\partial f}{\partial x}+\left(e \frac{d \Phi}{d x}-\mu \nabla B\right) \frac{\partial f}{\partial \mathbf{v}_{\|}}=S_{t}(f)+S_{t}^{*}(f)+S_{t}^{\mathrm{ECR}}(f),
$$

where $\|$ and $\perp$ symbols denote the directions along and perpendicular to the magnetic field, respectively, $S_{t}(f)$ is the collision integral for elastic collisions with atoms, $S_{t}^{*}(f)$ is the collision integral for inelastic collisions with atoms, and $S_{t}^{\mathrm{ECR}}(f)$ is the integral describing electron interactions with the wave electric field in the resonance point. The EDF is normalized according to

$$
n(x)=\int f\left(\mathbf{v}_{\|}, \mu, x\right) d \mathbf{v}_{\|} \mathbf{v}_{\perp} d \mathbf{v}_{\perp} .
$$

Note that the distribution function $f\left(\mathbf{v}_{\|}, \mu\right) d \mathbf{v}_{\|} d \mu$ corresponds to a linear density of electrons along magnetic lines $n d S / d S_{0}$, where $d S$ is the area between neighboring magnetic surfaces. The magnetic flux is constant, so $B(x) d S(x)$ $=B_{0} d S_{0}$ and the linear density scales as $n B_{0} / B(x)$. Let us consider the case of a monotonically increasing magnetic field. Due to the conservation of the magnetic momentum and energy, particles with low energy cannot penetrate into the regions with higher magnetic field. In a currentless plasma one can expect that the linear density of particles along a magnetic tube is smaller there. Nevertheless, the volume density $n$ is uniform for isotropic EDF. The uniform Maxwell distribution function

$$
\begin{aligned}
f & =n\left(\frac{m}{2 \pi T_{e}}\right)^{3 / 2} \exp \left(-\frac{m \mathrm{v}^{2}}{2 T_{e}}\right) \\
& =n\left(\frac{m}{2 \pi T_{e}}\right)^{3 / 2} \exp \left(-\frac{m \mathrm{v}_{\|}^{2}+\mu B}{2 T_{e}}\right)
\end{aligned}
$$

is a solution of the kinetic equation (7) without collisions on the right-hand side and the density is uniform, independently of the magnetic field configuration.

Substituting the expression for the integral $S_{t}^{\mathrm{ECR}}(f)$, the kinetic equation (7) reads:

$$
\begin{aligned}
\mathbf{v}_{\|} \frac{\partial f}{\partial x}+\left(e \frac{d \Phi}{d x}-\mu \nabla B\right) \frac{\partial f}{\partial \mathbf{v}_{\|}}= & S_{t}(f)+S_{t}^{*}(f)+\mathbf{v}_{\|} \delta\left(x-x_{r}\right) \\
& \times \frac{\partial}{\partial \mu} D_{\mu} \frac{\partial f}{\partial \mu}
\end{aligned}
$$

Further simplifications are possible by averaging over the fast electron bouncing. The total energy $\varepsilon_{\|}=0.5 m \mathrm{v}_{\|}^{2}$ $-e \Phi(x)+\mu B(x)$ is conserved, so it is natural to perform a transformation from variables $\mathrm{V}_{\|}, \mu$ to the new variables: longitudinal energy $\varepsilon_{\|}$(for the particular case of an ECR discharge the total energy coincides with the longitudinal energy), and $\mu$. In new variables, Eq. (9a) reads 


$$
\begin{aligned}
\left.\mathbf{v}_{\|} \frac{\partial f}{\partial x}\right|_{\varepsilon}-S_{t}(f)= & S_{t}^{*}(f)+\mathbf{v}_{\|} \delta\left(x-x_{r}\right)\left(\frac{\partial}{\partial \mu}+\frac{B \partial}{\partial \varepsilon}\right) \\
& \times D_{\mu}\left(\frac{\partial}{\partial \mu}+\frac{B \partial}{\partial \varepsilon}\right) f,
\end{aligned}
$$

We consider a low-pressure discharge where the bounce frequency is higher than or comparable with the collision frequency. The opposite case can be easily studied by a twoterm approximation. We focus on the time scale of the EDF evolution, which is governed by the inelastic collision frequency $\nu^{*}$. In most gases $\nu^{*} \ll \nu$. As we shall see, the electron-wave interaction integral can be estimated as $\left|S_{t}^{*}(f)\right| \lesssim \nu^{*} f$ in the stationary state. This means that the terms on the left-hand side of Eq. (9b) are much larger than the terms on the right-hand side. The isotropic, uniform EDF in total energy equalizes the large terms on the left-hand side of Eq. (9b) to zero. Thus, when the conditions $\nu \gg \nu^{*}$ and $\mathrm{V} / L \gg \nu^{*}$ are applicable, the EDF satisfies $f(\varepsilon, x, t)$ $\approx F_{0}(\varepsilon)$. In other words, the total energy is practically conserved during electron bouncing between turning points and scattering in elastic collisions. The assumption that the EDF depends solely on the total electron energy $\varepsilon$ proves to be suitable for an adequately accurate description of the electron kinetics. In comparison to $F_{0}(\varepsilon)$, the coordinate-dependent parts of the EDF are small corrections of the order of $\left(\nu^{*} / \nu\right),\left(\mathrm{V} L / \nu^{*}\right)^{2}[26]$.

To find $F_{0}(\varepsilon)$ we need to perform averaging of Eq. (9a) over the coordinate and the velocity angle. To do so, it is natural to rewrite Eq. (9a) in an integral form

$$
\iiint\left(-S_{t}^{*}(f)\right) d V=-\oint \int \vec{\Gamma} d \vec{S}
$$

where $d V=B(x) d x d \mu d \mathbf{v}_{\|}$is the phase volume, $\vec{\Gamma}=\mathbf{v}_{\|} f \vec{e}_{x}$ $-\mathrm{v}_{\|} \delta\left(x-x_{r}\right) D_{\mu}\left(\vec{e}_{\mu}(\partial / \partial \mu)+\vec{e}_{\varepsilon}(\partial / \partial \varepsilon)\right) f$ is the flux of EDF across boundaries of the phase volume. We can integrate Eq. (10) over the phase volume limited by two neighboring values of total energy: $\varepsilon$ and $\varepsilon+d \varepsilon$. We assume, first, that the energy is lower than the wall potential, so that the electrons are trapped in a potential well between turning points corresponding to $\mu=0$ and $\mathrm{v}_{\|}=0: X_{ \pm}(\varepsilon)$, where $\varepsilon$ $=-e \Phi\left(X_{+}\right)$. The phase volume is

$$
\begin{aligned}
\Delta V & =\iiint \varepsilon_{\varepsilon<\varepsilon\left(x, \mu, \mathrm{v}_{I I}\right)<\varepsilon+d \varepsilon} B(x) d x d \mu d \mathbf{v}_{\|} \\
& =(d \varepsilon / m) \int_{X_{-}(\varepsilon)}^{X_{+}(\varepsilon)} \mathrm{v} d x,
\end{aligned}
$$

where $\mathrm{V}$ is the absolute value of the velocity: $\mathrm{V}$ $=\sqrt{2(\varepsilon+e \Phi(x)) / m}$. There are no fluxes across the coordinate boundaries $X_{ \pm}(\varepsilon)$, since the electrons are trapped. The only remaining flux is due to the diffusion in the energy space. Assuming $f(x, \mu, \varepsilon) \approx F_{0}(\varepsilon)$, we find

$$
\oint_{\varepsilon<\varepsilon\left(x, \mu, \mathrm{v}_{I I}\right)<\varepsilon+d \varepsilon} \vec{\Gamma} d \vec{S}=d \Gamma_{\varepsilon}(\varepsilon),
$$

where $\Gamma_{\varepsilon}(\varepsilon)=-\left\langle\mathrm{v} \bar{D}_{\varepsilon}\right\rangle\left[d F_{0}(\varepsilon) / d \varepsilon\right]$, and $\left\langle\mathrm{v} \bar{D}_{\varepsilon}\right\rangle$ is the energy diffusion coefficient averaged over the space-pitch scattering angle:

$$
\begin{aligned}
\left\langle\mathrm{v} \bar{D}_{\varepsilon}\right\rangle & =\iiint D_{\varepsilon}\left(x, \mu, \mathrm{v}_{\|}\right) \delta\left(\varepsilon-\varepsilon\left(x, \mu, \mathrm{v}_{\|}\right)\right) B d x d \mu d \mathbf{v}_{\|} \\
& \equiv \int_{X_{-}(\varepsilon)}^{X_{+}(\varepsilon)} d x \int_{0}^{\mathrm{v}_{\| \max }} D_{\varepsilon}\left(x, \mu\left(\varepsilon, \mathrm{v}_{\|}\right), \mathbf{v}_{\|}\right) d \mathbf{v}_{\|}
\end{aligned}
$$

where $\mathrm{V}_{\| \max } \equiv \mathrm{v}=\sqrt{2(\varepsilon+e \Phi(x)) / m}, B \mu\left(\varepsilon, \mathrm{V}_{\|}\right)=\varepsilon+e \Phi(x)$ $-m \mathrm{v}_{\|}^{2} / 2$, and $D_{\varepsilon}=\mathrm{v}_{\|} \delta\left(x-x_{r}\right) B^{2} D_{\mu}$.

As a result we obtain the traditional kinetic averaged equation in the nonlocal approach [27], $\overline{\left\langle S_{t}^{*}(f)\right\rangle}$ $+\overline{\left\langle S_{t}^{\mathrm{ECR}}(f)\right\rangle}=0$ where double averaging is produced according to $\langle\bar{G}\rangle=\int_{x(\varepsilon)}^{x_{+} \varepsilon} d x \int_{0}^{\mathrm{v}_{\| \max }} d \mathbf{v}_{\|}$. We assume that the resonance point coincides with the coordinate of the potential minimum, the opposite case will be discussed later. The averaging of $\overline{\left\langle S_{t}^{\mathrm{ECR}}(f)\right\rangle}$ is especially simple for the ECR discharge due to the $\delta$ function in Eq. (5). Integration over $\mathbf{V}_{\|}$ and $x$ gives the simple answer

$$
\overline{\left\langle S_{t}^{\mathrm{ECR}}(f)\right\rangle}=(d / d \varepsilon)\left\langle\mathrm{v} \bar{D}_{\varepsilon}\right\rangle(d f / d \varepsilon),
$$

and the averaged kinetic equation reads

$$
\begin{aligned}
& \frac{d}{d \varepsilon}\left(\overline{\mathrm{V} D_{\varepsilon}}(\varepsilon) \frac{d F_{0}(\varepsilon)}{d \varepsilon}\right) \\
& \quad=\sum_{k} \overline{\mathrm{V} \nu_{k}^{*}}(\varepsilon) F_{0}(\varepsilon)-\sum_{k} \overline{\mathrm{V} \nu_{k}^{*}}\left(\varepsilon+\varepsilon_{k}^{*}\right) F_{0}\left(\varepsilon+\varepsilon_{k}^{*}\right),
\end{aligned}
$$

where

$$
\langle\mathrm{v} \bar{D}\rangle_{\varepsilon}=\frac{4 \pi}{3} \frac{\left(e E_{\perp} \varepsilon\right)^{2}}{m\left|\frac{d \omega_{B}}{d x}\right| \sqrt{2 m \varepsilon}},
$$

and the upper bar denotes only averaging in coordinate, since the averaging of inelastic frequency in velocity angle is trivial:

$$
\left\langle\overline{\nu_{k}^{*}}\right\rangle=\int_{X_{-}(\varepsilon)}^{X_{+}(\varepsilon)} d x \int_{0}^{\mathrm{v}_{\| \max }} d \mathrm{v}_{\|} \nu_{k}^{*}=\int_{X_{-}(\varepsilon)}^{X_{+}(\varepsilon)} d x \mathrm{v} \nu_{k}^{*} \equiv \overline{\mathrm{v} \nu_{k}^{*}}
$$

\section{MONTE CARLO SIMULATION OF THE ELECTRON VELOCITY DISTRIBUTION FUNCTION IN THE ECR DISCHARGES}

To verify the analytical results, we have developed a MC model of the ECR discharge, which is one dimensional in space and two dimensional in velocity. The electrons are moving in the stationary electric field with potential $\Phi(x)$ and magnetic field $B(x)$ through the discharge of a length $L$. There is an electron cyclotron resonance zone in the middle of the discharge, where the electrons experience a change $\Delta \varepsilon_{\perp}$ (a 'kick') in the perpendicular energy according to Eq. (3), with a random phase $\theta$.

The electrons are either absorbed or reflected at the boundaries depending on the magnitude of their parallel kinetic energy compared to the prescribed wall potential. All cross sections were taken in the form $\sigma\left(\vartheta, E, E_{1}\right)$ $=\Pi\left(\vartheta, E_{1}\right) \sigma(E)$, where $E$ is the electron energy before col- 
lision $\sigma(E)$ is the total cross section, $E_{1}$ is the kinetic energy after the collision (i.e., with the energy loss deducted) in $\mathrm{eV}$, and the dependence on the scattering angle $\vartheta$ was adopted from Ref. [28] in a form

$$
\Pi\left(\vartheta, E_{1}\right)=\frac{E_{1}}{2 \ln \left(1+E_{1} / 2\right)\left(E_{1} \sin ^{2} \vartheta / 2+l\right)} .
$$

The elastic and excitation collisions of the electrons with argon atoms are incorporated into the model. The data from Ref. [29] were fitted to obtain the total elastic cross section in $\mathrm{cm}^{2}$ :

$$
\sigma_{e l}(E)=\left\{\begin{array}{l}
1.5 \times 10^{-16}, \text { for } E \leqslant 0.08 \mathrm{eV} \\
10^{-16} \exp \left(y_{1}\right) \text { for } 0.08 \mathrm{eV} \leqslant E \leqslant 16 \mathrm{eV} \\
y_{2} \text { for } 16 \mathrm{eV} \leqslant E \leqslant 100 \mathrm{eV} \\
5.901 \times 10^{-15} \times E^{-0.5443} \text { for } E \geqslant 100 \mathrm{eV}
\end{array}\right.
$$

where

$$
\begin{aligned}
y_{2}=10^{-15}[ & 4.658+E\left\{-0.2073+10^{-3} E\left\{4.506+10^{-2}\right.\right. \\
\times E[- & \left.\left.\left.\left.4.974+10^{-2} E\left(2.684-5.613 \times 10^{-3} E\right)\right\}\right]\right\}\right], \\
y_{1}= & (0.1655+x\{1.613+x[-0.07181 \\
& +x(-0.3825+x\{0.1339+x[0.04361 \\
& +x(-0.01297-x 0.001541)]\})]\}),
\end{aligned}
$$

$x=\ln (E)$; and $E$ is the electron kinetic energy in $\mathrm{eV}$. The fit for $E>100 \mathrm{eV}$ is from Ref. [30].

The excitation only to one level of $11.5 \mathrm{eV}$ is considered using the cross section in a simplified form given by

$$
\sigma_{\text {exc }}(E)=\left\{\begin{array}{c}
0 \quad \text { for } E \leqslant 11.5 \mathrm{eV} \\
7 \times 10^{-18}(E-11.5 \mathrm{eV}) \mathrm{cm}^{2} \mathrm{eV} \\
\text { for } 11.5 \mathrm{eV} \leqslant E \leqslant 20 \mathrm{eV} \\
5.95 \times 10^{-17} \mathrm{~cm}^{2} \text { for } E \geqslant 20 \mathrm{eV}
\end{array}\right.
$$

The discharge length is divided into a regularly spaced grid. The electrostatic potential and magnetic field are defined on the grid. A test electron is started with initial parallel and perpendicular energies of $1 \mathrm{eV}$. We used a spatial MC technique, instead of the conventional temporal one; see, e.g., Ref. [31]. It implies that one step of the simulation consists of moving the test electron from a grid point to the next spatial grid point. Next an appropriate bin of the distribution function in the total energy $(\varepsilon)$ and in the first invariant $(\mu)$ is incremented by unity, and the elapsed time is increased by the time of flight between the grid points. The collisions are treated by the null-collision technique. If the time remaining until the next collision is smaller than the time of flight between the grid points, the position of the collision is found, the kind of collision is determined, the velocity scattering and energy loss are performed upon the test electron, and a new collision time is sampled from the exponential distribution using maximum collision frequency and a random number. The integration then continues from the position of collision. When the electron is lost at the boundary, a new electron is started with the initial position a)

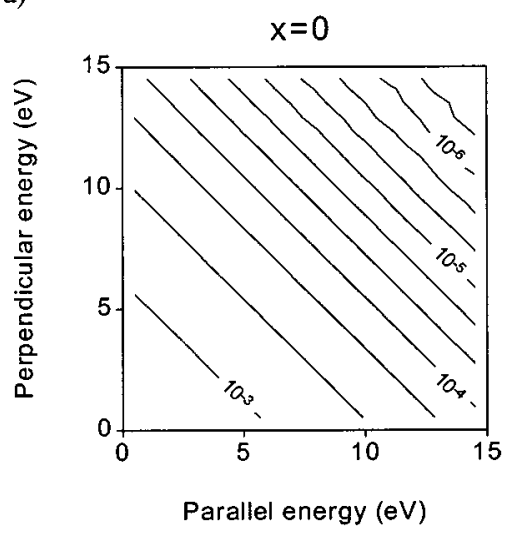

b)

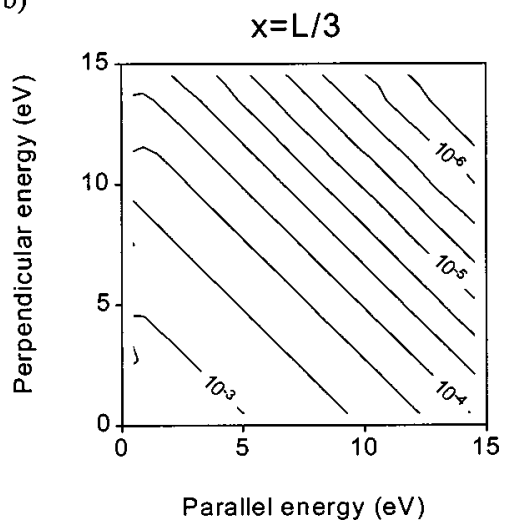

FIG. 1. The EDF as a function of the perpendicular kinetic energy $\left(m v_{\perp}^{2} / 2=\mu B\right)$ and parallel kinetic energy $m \mathrm{v}_{\|}^{2} / 2$ at two locations in the discharge; (a) $x=0$, and (b) $x=L / 3 . L=0.2 \mathrm{~m}$, the electric field in the resonance is $0.7 \mathrm{~V} / \mathrm{cm}$, the potential $-U_{0}(x / 2 L)^{2}, \quad U_{o}=5 \mathrm{~V}, B(x)=B_{0}\left[1+(2 / \pi) \arctan \left(-x / x_{0}\right)\right], \quad B_{0}$ $=0.0875 \mathrm{~T}$, and $x_{0}=5 \mathrm{~cm}$.

and energies determined using the distribution function recorded so far. The procedure is continued for a total time long enough to get obtain sufficient statistics in the distribution function over the range of energies of interest. Finally, the distribution function in the total energy and the first invariant are recalculated to the velocity distribution function as functions of parallel and perpendicular energy. It is expected that our spatial MC technique is faster than the temporal MC technique (steps in time rather than space are made) for large mean free paths, when collisions within one step on the spatial grid are rare. In the opposite case, the traditional temporal technique is more appropriate.

\section{VERIFICATION OF ELECTRON BOLTZMANN KINETIC EQUATION AVERAGED OVER FAST ELECTRON BOUNCING AND PITCH-ANGLE SCATTERING BY COMPARISON WITH MC RESULTS}

Since we intend to analyze only electron kinetics in this section, we restricted ourselves in these simulations to the case of a fixed ambipolar potential $\Phi(x)$ and a given wave electric field in the resonance point. First we check the assumption of an isotropic EDF. The discharge parameters are of width $L=0.2 \mathrm{~m}$; the potential is modeled in the parabolic forms $\Phi(x)=-U_{0}(2 x / L)^{2}$ and $U_{0}=5 \mathrm{~V}$, unless stated otherwise. The magnetic field is taken in the form $B(x)$ 
a)

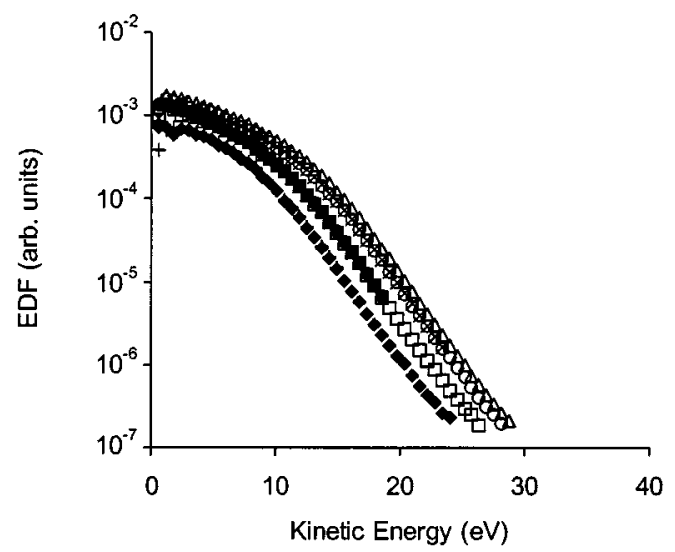

b)

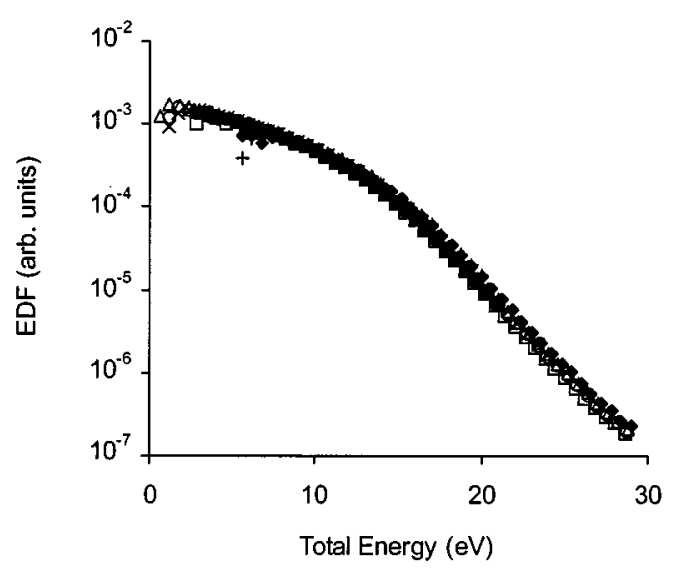

FIG. 2. The EDF as a function of (a) kinetic energy $\left[m\left(\mathrm{~V}_{\perp}^{2}\right.\right.$ $\left.\left.+\mathrm{v}_{\|}^{2}\right) / 2\right]$ and (b) total energy $\left[m\left(\mathrm{v}_{\perp}^{2}+\mathrm{v}_{\|}^{2}\right) / 2\right]-e \Phi(x)$ at various locations in the discharge. The discharge parameters are the same as in Fig. 1.

$=B_{0}(1+(2 / \pi) \arctan (-x / 0.1 \mathrm{~m}))$, where $B_{0}=875 \mathrm{G}$. In some runs for the sake of comparison, we use a homogeneous magnetic field $B=875 \mathrm{G}$, but with the same value of velocity kick as for the inhomogeneous magnetic field. An argon pressure or $p=1 \mathrm{~m}$ Torr was assumed, unless stated otherwise.

In Fig. 1 the EDF's are shown as functions of the perpendicular kinetic energy $\left(m \mathrm{v}_{\perp}^{2} / 2=\mu B\right)$ and parallel kinetic energy $m \mathrm{v}_{\|}^{2} / 2$ at the discharge center and at $x=L / 3$. One can see that the EDF's are perfectly isotropic at the shown locations. As discussed above, this implies that the EDF is isotropic everywhere. The results of MC calculations confirm this finding. In Fig. 2, EDF's at different spatial points are shown. All EDF's coincide with each other after being redrawn as a function of the total energy.

The EDF's calculated by both FM and Monte Carlo methods are presented jointly in Fig. 3 for comparison. The agreement between FM and MC methods appears to be reasonable. In Fig. 3 one can see that the isotropic EDF does not depend on the magnetic field distribution, provided that the velocity kick in the resonance is the same, as follows from Eq. (12).

We also checked if the model of diffusion in energy is correct. For this purpose we calculated the energy diffusion coefficient $\left\langle D_{\varepsilon}\right\rangle=\left\langle(\Delta \varepsilon)^{2} / \Delta t\right\rangle$ with the use of the MC tech-

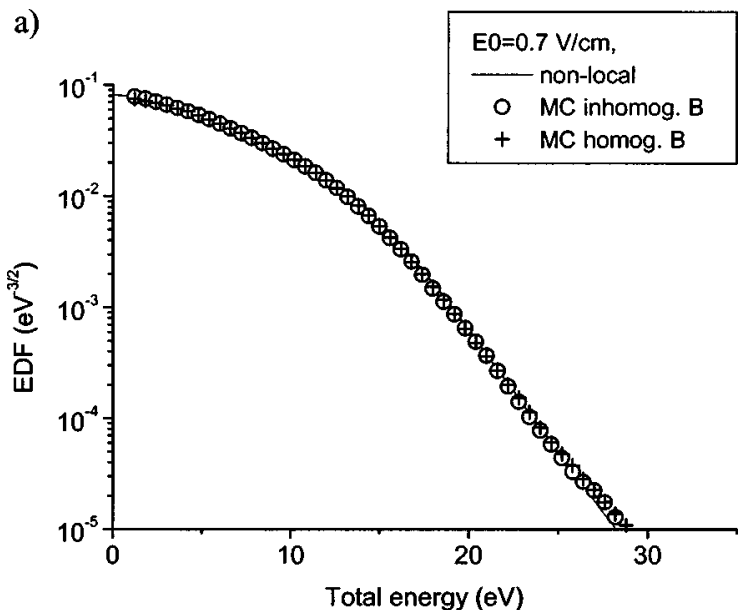

b)

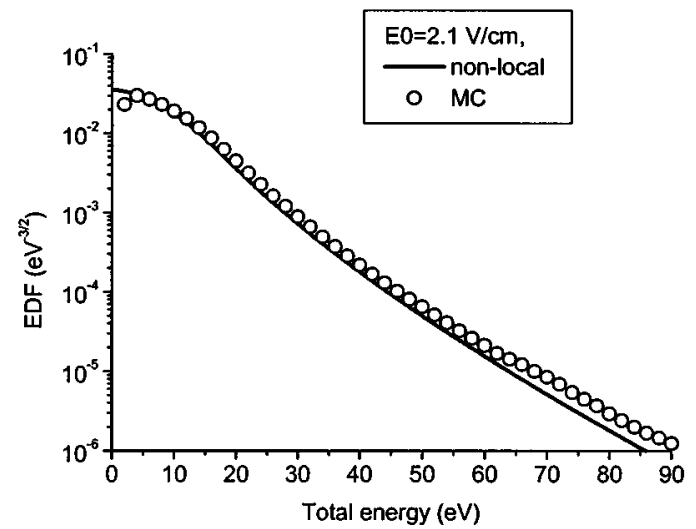

FIG. 3. The EDF's calculated from the averaged kinetic equation (solid lines) and by the MC method (symbols) as functions of the total energy. The argon pressure $p=1 \mathrm{~m}$ Torr, no wall losses are accounted for, the given potential profile is $-\Phi(x)=U_{0}(2 x / L)^{2}$, and the discharge width $L=0.2 \mathrm{~m}$. Circles correspond to the magnetic field $B(x)=B_{0}\left[1+(2 / \pi) \arctan \left(-x / x_{0}\right)\right], B_{0}=0.0875 \mathrm{~T}, x_{0}$ $=10 \mathrm{~cm}$, and crosses correspond to a homogeneous magnetic field and the same velocity kick. (a) The electric field in the resonance is $E_{\perp}=0.7 \mathrm{~V} / \mathrm{cm}$. (b) $E_{\perp}=2.1 \mathrm{~V} / \mathrm{cm}$.

nique by averaging the ratio of the square of the energy change $\Delta \varepsilon$ and time $\Delta t$ over the electron ensemble. The time $\Delta t$ was chosen large compared with the bounce time and the time between collisions $\Delta t \gg L / \mathrm{V}$ and $\nu$, but not too large, so that the energy change during time $\Delta t$ is $\Delta \varepsilon(\Delta t) \ll \varepsilon$. For no electric field present in the plasma (corresponding to a uniform plasma density)

$$
\left\langle D_{\varepsilon}\right\rangle=\frac{\left\langle\mathrm{v} \bar{D}_{\varepsilon}\right\rangle}{L \mathrm{v}}=\frac{2 \pi \varepsilon}{3 m L} \frac{\left(e E_{\perp}\right)^{2}}{\left|\frac{d \omega_{e c}}{d x}\right|} .
$$

In Fig. 4 one can see that the analytical expression predicts the value of $\left\langle D_{\varepsilon}\right\rangle$ fairly well.

\section{ANALYTICAL SOLUTION FOR THE EDF IN THE LOSS CONE}

In this section we shall provide a general solution for the EDF in the loss cone for any discharge. Simulations of the 


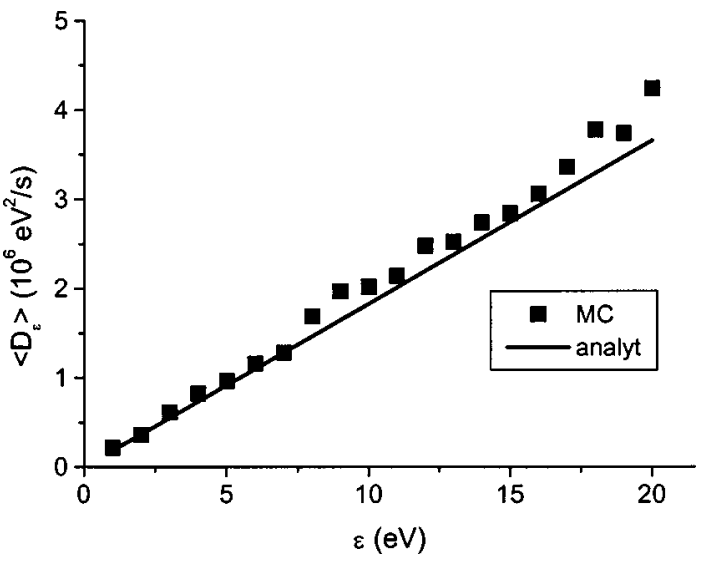

FIG. 4. The energy diffusion coefficient $\left\langle\bar{D}_{\varepsilon}\right\rangle=\left\langle(\Delta \varepsilon)^{2} / \Delta t\right\rangle$ as a function of energy calculated by the MC method for a homogeneous potential and the analytical expression

$$
\left\langle\bar{D}_{\varepsilon}\right\rangle=\frac{2 \pi}{3} \frac{\left(e E_{\perp}\right)^{2} \varepsilon}{m\left|\frac{d \omega_{B} \mid}{d x}\right| L} .
$$

$B(x)=B_{0}\left[1+(2 / \pi) \arctan \left(-x / x_{0}\right)\right], \quad B_{0}=0.0875 \mathrm{~T}, \quad$ and electric field in the resonance is $E_{\perp}=0.7 \mathrm{~V} / \mathrm{cm}$.

EDF in the loss cones have been described in many papers; see, e.g., Ref. [31,37]. However, to our knowledge no analytical solution for the EDF in the loss cone was found earlier. In this section we provide this analytical solution, and also show how the wall losses should be incorporated into Eq. (12) for the main part of the EDF. We start the discussion without taking the magnetic field into account, and at the end generalize the result for the presence of an inhomogeneous magnetic field. We also assume first that the electrostatic potential is symmetric in the left and right parts of the discharge. The generalization for an asymmetric potential is obvious.

Electrons can escape if their energy is higher than the wall potential $\varepsilon>-e \Phi_{w}$. The electrons with energies $-e \Phi_{w}$ $<\varepsilon<-e \Phi_{w}+(2-3) T_{\text {tail }}$, where $T_{\text {tail }}=-(d \ln f / d \varepsilon)_{\varepsilon=-e \Phi_{w}}^{-1}$ is a characteristic energy scale at the tail of the EDF, determine the electron flux to the wall and are of primary interest. Obviously, the flux of electrons with $\varepsilon>-e \Phi_{w}$ $+(2-3) T_{\text {tail }}$ is negligible. Typically $T_{\text {tail }} \sim 1-2 \mathrm{eV}$ [32], and for most cases the wall potential is slightly above the ionization potential $(I):-e \Phi_{w} \sim I$. This implies that $T_{\text {tail }}$ $\ll-e \Phi_{w}$. The wall potential can be below the ionization potential in the case of high pressure discharges, where the electron undergoes many collisions before reaching the wall, i.e., there is a high probability of ionization event for a single pass of an electron across the discharge. Then the wall potential above the ionization potential is not anymore a necessary condition for a self-sustained discharge [32].

Electrons with a given total energy, which can escape to the wall, form a loss cone in the phase space. They are characterized by a positive velocity projection perpendicular to the walls $\mathrm{v}_{x}$ (wall) $>0$ and by a parallel energy $\varepsilon_{x} \equiv m \mathrm{v}_{x}^{2} / 2$ $-e \Phi(x)>-e \Phi_{w}$. The latter condition is equivalent to a perpendicular energy $\varepsilon_{\perp} \equiv m\left(\mathrm{v}_{y}^{2}+\mathrm{v}_{z}^{2}\right) / 2=\varepsilon-\varepsilon_{x}$ being small, i.e., $\varepsilon_{\perp}<\varepsilon+e \Phi_{w}$. Thus from electrons with a total energy large enough to overcome the wall potential i.e., $-e \Phi_{w}<\varepsilon$ $<-e \Phi_{w}+(2-3) T_{\text {tail }}$, only a small fraction for which $\varepsilon_{\perp}$

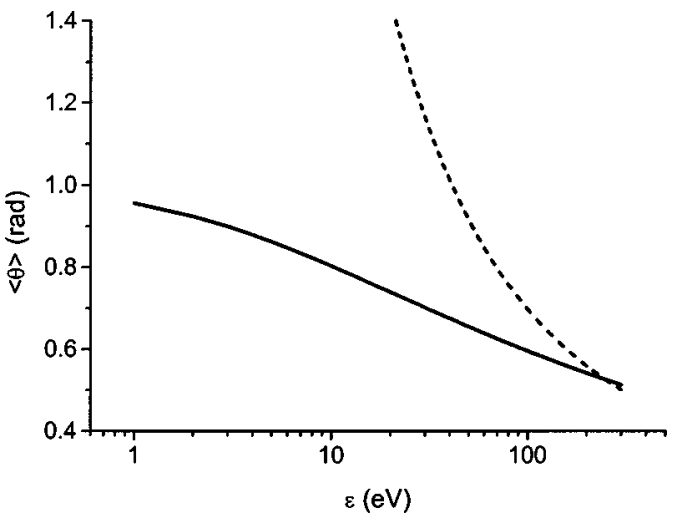

FIG. 5. Average scattering angle $\langle\vartheta\rangle=\frac{1}{2} \int_{0}^{\pi} \Pi(\vartheta, \varepsilon) \sin \vartheta d \vartheta$ as a function of energy for differential cross section taken in the form

$$
\Pi(\vartheta, \varepsilon)=\frac{\varepsilon}{2 \ln (1+\varepsilon / 2)\left(\varepsilon \sin ^{2} \vartheta / 2+1\right)} .
$$

The solid line corresponds to the calculated integral, and the dashed line to the analytical limit at high energies $\varepsilon \gg 1\langle\vartheta\rangle \rightarrow(1$ $+\pi / \sqrt{\varepsilon}) /[2 \ln (1+\varepsilon / 2)]$

$<(2-3) T_{\text {tail }}$ can actually escape. The fact that the fraction is small follows from the assumption $T_{\text {tail }} \ll-e \Phi_{w}$. In other words, the loss cone is small. The assumption of a small loss cone allows us to find an analytical solution for the EDF that accounts for wall losses.

To find the EDF analytically in the loss cone we shall assume that the differential cross section has no singularity at small angles. This is correct for an electron kinetic energy of the order of the ionization potential. Figure 5 depicts the average scattering angle as a function of the energy for argon [the differential cross sections are given by Eq. (13)]. One can see that average scattering angle is large, $\langle\vartheta\rangle \sim 0.5 \mathrm{rad}$, for $\varepsilon \sim I$. For high energies $\varepsilon \gg I$, the differential cross section eventually should approach Coulomb scattering, so the approximation we used for $\varepsilon \sim I$ is not applicable. In the following we therefore assume the small angle scattering is not important and can be neglected. If important, the small angle scattering can be incorporated by describing the collision integral as diffusion in the velocity angle.

The distribution function outside the loss cone (OLC) is quite different from the EDF inside the loss cone (LO) $f_{\mathrm{LC}}$. The main processes that form the EDF's inside and outside the LC are elastic scattering and spatial displacement, so that other processes (inelastic collisions and diffusion in energy) can be neglected. The collisional integral with neutrals can be written in the form: $S_{t}(f)=\int\left(f^{\prime}-f\right) \mathrm{v}(d \sigma / d \Omega) d \Omega$, where $f^{\prime}$ is the EDF of electrons before scattering to a given velocity $\vec{v}$. The EDF is determined by the equation

$$
\left.\mathrm{v}_{x} \frac{\partial f}{\partial x}\right|_{\varepsilon}=\int\left(f^{\prime}-f\right) \vee \frac{d \sigma}{d \Omega} d \Omega .
$$

The EDF outside the loss cone is close to isotropic, and is only a function of total energy only $F_{0}$. Indeed the electrons outside the loss cone can be scattered into the loss cone due to the elastic collisions, and consequently be lost. But since the loss cone is assumed to be small, the electrons will remain more frequently in the OLC region rather than leave to the LC. This results in an isotropic EDF. We can estimate 
the deviation from the isotropic EDF outside the loss cone $\delta f \equiv f-F_{0}$ from Eq. (14). The collision integral reads $\int\left(f^{\prime}-f\right) \mathrm{v}(d \sigma / d \Omega) d \Omega=\int_{\mathrm{olc}} f^{\prime} \mathrm{\vee}(d \sigma / d \Omega) d \Omega+\int_{c} f^{\prime} \mathrm{\vee}(d \sigma /$ $d \Omega) d \Omega-\vee \sigma f$, where the first integral is over the region OLC and the second one inside the LC only. For an isotropic $\mathrm{EDF}, \quad \operatorname{St}\left(F_{0}\right)=0$ and $\int_{\mathrm{olc}} F_{0}^{\prime} \mathrm{v}(d \sigma / d \Omega) d \Omega+\int_{c} F_{0}^{\prime} \mathrm{v}(d \sigma /$ $d \Omega) d \Omega=\vee \sigma F_{0}$. Substituting $f=F_{0}+\delta f$ outside the loss cone and $f=f_{l c}$ in the loss cone, we find the collision integral $\int_{c}\left(f_{l c}-F_{0}\right)^{\prime} \mathrm{v}(d \sigma / d \Omega) d \Omega-\mathrm{v} \sigma \delta f+\int_{\text {olc }} \delta f^{\prime} \mathrm{v}(d \sigma /$ $d \Omega) d \Omega$. From this equation one can estimate that $\delta f$ $\lesssim\left(\Delta_{l c} \Omega / 4 \pi\right) F_{0} \ll F_{0}$ is small, since the loss cone is assumed to be small, $\Delta_{l c} \Omega / 4 \pi \ll 1$.

The relation between an EDF OLC and one in the LC can be found from Eq. (14). The main income term of the EDF in the loss cone is due to the pitch-angle scattering from the outside of the loss cone into the loss cone, which can be estimated as $\int_{\text {OLC }} f^{\prime}(d \sigma / d \Omega) \vee d \Omega \approx F_{0} \vee \sigma$, since the loss cone is small, and the integral over any isotropic function is $\int f^{\prime}(d \sigma / d \Omega) \vee d \Omega \approx \int f(d \sigma / d \Omega) \vee d \Omega$. As a result, Eq. (14) simplifies to

$$
\begin{gathered}
\left.\mathrm{v}_{x} \frac{\partial f_{l c}}{\partial x}\right|_{\varepsilon}=-\nu\left(f_{l c}-F_{0}\right), \\
f_{l c}(x=-L / 2)=0 \text { for } \mathrm{v}_{x}>0,
\end{gathered}
$$

and

$$
f_{l c}(x=L / 2)=0 \text { for } \mathrm{v}_{x}<0,
$$

where $\nu \equiv \vee \sigma$. Equation (15) has obvious solutions

$$
\begin{gathered}
f_{l c}\left(\varepsilon, \mathrm{v}_{x}, x\right)=F_{0}\left[1-\exp \left(-\int_{-L / 2}^{x} \frac{\nu d x}{\mathrm{v}_{x}}\right)\right] \quad \text { for } \mathrm{v}_{x}>0 \\
f_{l c}\left(\varepsilon, \mathrm{v}_{x}, x\right)=F_{0}\left[1-\exp \left(-\int_{x}^{L / 2} \frac{\nu d x}{\left|\mathrm{v}_{x}\right|}\right)\right] \text { for } \mathrm{v}_{x}<0
\end{gathered}
$$

A similar solution was found for the electron-electron collision integral in Ref. [33]. If we sample the EDF for both velocity directions; $f_{l c \pm}\left(\varepsilon,\left|\mathrm{v}_{x}\right|, x\right)=\left[f_{0}\left(\varepsilon, \mathrm{v}_{x}, x\right)+f_{0}(\varepsilon\right.$, $\left.\left.-\mathrm{v}_{x}, x\right)\right] / 2$, we find

$$
f_{l c \pm}\left(\varepsilon,\left|\mathrm{v}_{x}\right|, x\right)=F_{0}\left[1-\exp \left(-\int_{0}^{L / 2} \frac{\nu d x}{\left|\mathrm{v}_{x}\right|}\right) \cosh \left(\int_{0}^{x} \frac{\nu d x}{\left|\mathrm{v}_{x}\right|}\right)\right] \text {. }
$$

If the mean free path is larger than the gap $\nu L / \mathrm{V} \ll 1$, the EDF in the LC is strongly depleted $f_{l c} \sim(\nu L / v) F_{0} \ll F_{0}$. In the opposite case of a small mean free path $\nu L / \mathrm{v}_{x} \gg 1$, the depletion in the LC is small $f_{l c} \approx F_{0}$ due to the fast scattering in elastic collisions. At the wall $f_{l c \pm}=F_{0} / 2$ due to the absence of electrons with velocity directed from the wall toward the plasma. The loss cone is empty at the wall and gets filled with electrons at distances of the order of the mean free path according to Eq. (16c).

Figure 6 depicts EDF's outside and inside the loss cone calculated by the MC code. One can see that the theoretical prediction for a uniform EDF outside the loss cone is reasonable within $20 \%$, which corresponds to the approximation of a small loss cone $\Delta_{l c} \Omega / 4 \pi \ll 1$. In Fig. 6, the EDF's practi-
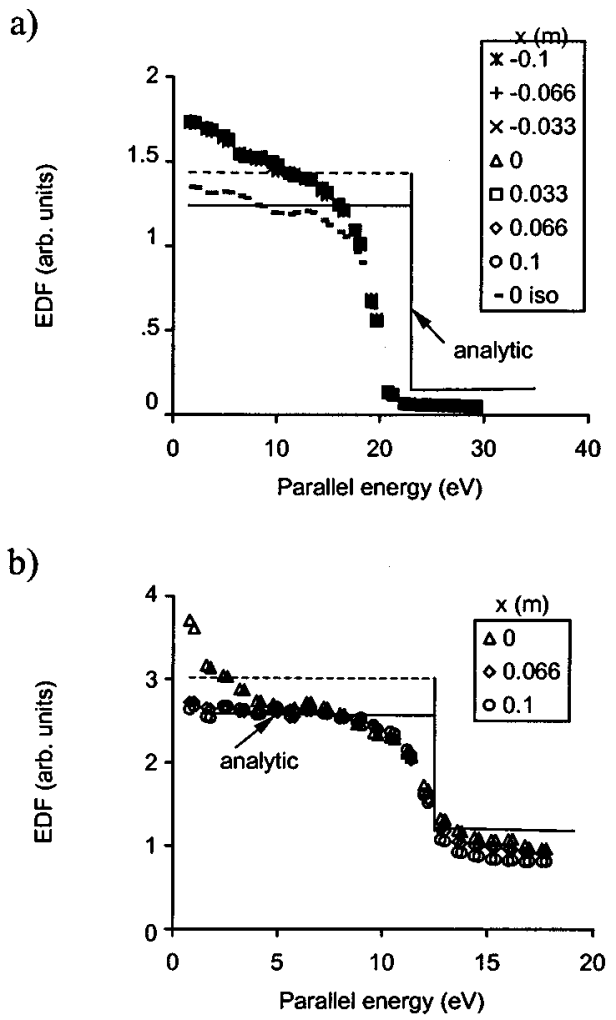

c)

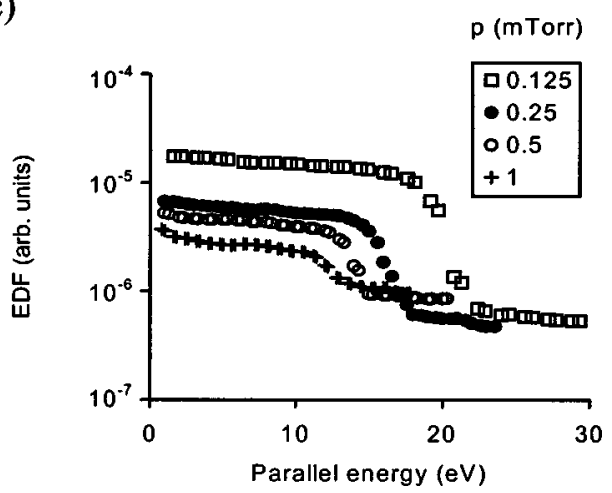

FIG. 6. EDF $f_{l c}\left(\varepsilon,\left|\mathrm{v}_{x}\right|, x\right)$ at given total energy $\varepsilon=-1.5 e \Phi_{w}$ as a function of the parallel energy at various coordinates. Solid lines correspond to the theoretical estimate $f_{l c}\left(\varepsilon,\left|\mathbf{v}_{x}\right|, x=0\right)=f_{0}[1$ $\left.-\exp \left(-\int_{0}^{L / 2}\left(\mathrm{v} \nu d x / \mathrm{v}_{x}\right)\right)\right]$, and dashed lines to the estimate with the total cross section replaced with the cross section of scattering outside the loss cone: $\sigma \rightarrow \sigma_{\mathrm{OLC}}=\int_{\mathrm{OLC}}(d \sigma / d \Omega) / d \Omega$ $=\sigma(E) \int_{0}^{v_{x} \min / \nu} \Pi(\vartheta, E) d \cos \vartheta$; iso stands for isotropic cross section. (a) $p=0.125 \mathrm{~m}$ Torr, (b) $p=1 \mathrm{~m}$ Torr. (c) Distribution functions at discharge center. The wall potentials are $-\Phi_{w}=20.6 \mathrm{eV}$ for $p$ $=0.125 \mathrm{~m}$ Torr, $-\Phi_{w}=16.4 \mathrm{eV}$ for $p=0.25 \mathrm{~m}$ Torr, $-\Phi_{w}$ $=14.2 \mathrm{eV}$ for $p=0.5 \mathrm{~m}$ Torr, and $-\Phi_{w}=12.4 \mathrm{eV}$ for $p$ $=1 \mathrm{~m}$ Torr.

cally do not depend on the form of the differential cross sections, also according to the predictions of the theory, as follows from Eq. (16c). The EDF in the LC is spatially uniform: $f_{l c \pm}=F_{0} \int_{0}^{L / 2}\left(\nu d x /\left|\mathrm{v}_{x}\right|\right)$ for $\int_{0}^{L / 2}\left(\nu d x / \mid \mathrm{v}_{x}\right) \ll 1$. At $P$ $=0.125 \mathrm{~m}$ Torr, $\int_{0}^{L / 2}\left(\nu d x /\left|\mathrm{v}_{x}\right|\right)=0.066 \ll 1$, and the EDF's obtained in the MC simulation at various positions coincide with each other. At $P=1 \mathrm{~m}$ Torr, $\int_{0}^{L / 2}\left(\nu d x /\left|\mathrm{v}_{x}\right|\right)=0.80 \sim 1$, and the EDF's in the LC at the discharge center are larger than those at the periphery of the discharge. 
The analytical expression for the ratio of the EDF's outside and inside the loss cone, $f_{l c \pm}(x=0) / F_{0}=[1$ $\left.-\exp \left(-\int_{0}^{L / 2}\left(\nu d x /\left|\mathrm{v}_{x}\right|\right)\right)\right]$ underestimates the value obtained in the MC approach. This is probably because of the assumption of a small loss cone: it was assumed that an electron leaves the loss cone after scattering in any elastic collision. A more comprehensive theory should be developed for a more thorough description. For an approximate accounting of this fact, we can change the total cross-section to the crosssection of scattering only outside the loss cone:

$$
\begin{aligned}
\sigma & =\int(d \sigma / d \Omega) d \Omega \rightarrow \sigma_{\mathrm{olc}} \\
& =\int_{\mathrm{olc}}(d \sigma / d \Omega) d \Omega \\
& =\sigma(E) \int_{0}^{v_{x} \min } / \mathrm{v} \Pi(\vartheta, E) d \cos \vartheta,
\end{aligned}
$$

where $\left(m \mathrm{v}_{x \min }^{2} / 2\right)-e \Phi(x)=-e \Phi_{w}$. In Fig. 6 one can see that such substitution (compare dashed lines with solid ones) improves the agreement of MC results with this theory.

Knowing the EDF in the loss cone, the average frequency of the electron losses to the walls, $\nu_{\text {escape }}(\varepsilon)$, with a total energy $\varepsilon$, can be found. First we consider the case with no electric field present in the plasma bulk, and then discuss the general situation. Integration of the flux $\mathrm{v}_{x} f_{l c}(x=L)$ over all possible velocity directions at the wall $\int_{-\mathrm{v}}^{\mathrm{v}} d \mathrm{v}_{x} / 2 \mathrm{v}$ yields the average electron flux $\Gamma_{w}(\varepsilon)$ of electrons with a given total energy $\varepsilon$ :

$\Gamma_{w}(\varepsilon)=2 \int_{\mathrm{v}_{x \min }}^{\mathrm{v}} \frac{d \mathrm{v}_{x}}{2 \mathrm{v}} \mathrm{v}_{x} F_{0}\left[1-\exp \left(-\int_{-L / 2}^{L / 2} \frac{\nu d x}{\mathrm{v}_{x}}\right)\right]$.

where $\left(m \mathrm{v}^{2} / 2\right)-e \Phi(L / 2)=\varepsilon$ is the maximum velocity near the wall in front of the sheath; $\left(m \mathrm{v}_{x \min }^{2} / 2\right)-e \Phi(L / 2)=$ $-e \Phi_{w}$ is the minimum velocity of electrons capable of overcoming the wall potential and escaping and the factor of 2 accounts for the two walls. The average escape frequency is given by the ratio of the flux to the total electron density in the plasma slab of a width $L, \nu_{\text {escape }} \equiv \Gamma_{w}(\varepsilon) /\left(F_{0} L\right)$ :

$\nu_{\text {escape }}(\varepsilon)=\int_{0_{x \min }}^{\mathrm{v} L} \frac{d \mathrm{v}_{x}}{\mathrm{v} L} \mathrm{v}_{x}\left[1-\exp \left(-\int_{-L / 2}^{L / 2} \frac{\nu d x}{\mathrm{v}_{x}}\right)\right]$.

In Eq. (18), losses at both walls are accounted for. If the mean free path is smaller than the gap, then $f_{l c} \approx F_{0}$ and $\nu_{\text {escape }}(\varepsilon) \approx \int_{\mathrm{v}_{x \text { min }}}^{\mathrm{v}}\left(d \mathrm{v}_{x} / \mathrm{v} L\right) \mathrm{v}_{x}=\left(\varepsilon+e \Phi_{w}\right) / m \mathrm{v} L$, the average flux to the wall is $\left.\Gamma_{w}=\left(\varepsilon+e \Phi_{w}\right) / 2 m \mathrm{v}\right) F_{0}$. In the two-term approximation this corresponds to the boundary condition at the plasma sheath boundary in the form

$$
-\left.\mathrm{v} D_{x} \frac{\partial F_{0}(\varepsilon, x)}{\partial x}\right|_{x=L}=\frac{\varepsilon+e \Phi_{w}}{2 m} F_{0}(\varepsilon, L) .
$$

In Eq. (19), losses at the nearest wall are only accounted for the factor $\frac{1}{2}$. In Refs. [31,34], approximate boundary conditions were used, which can be deduced from Eq. (17) if the velocity projection is substituted for the absolute value of velocity $\mathrm{V}_{x} \rightarrow \mathrm{V}$. Note that the boundary condition (19) is more appropriate as the correct one, and even more simple. In the opposite case of a large mean free path, $\nu_{\text {escape }}(\varepsilon)$ $=\int_{\mathrm{v}_{x \min }}^{\mathrm{v}} d \mathrm{v}_{x} /(\mathrm{v} L) \mathrm{v}_{x} \int_{-L / 2}^{L / 2}\left(\nu d x / \mathrm{v}_{x}\right) \sim \nu\left(\Delta_{e c} \Omega / 4 \pi\right)$ is propor- tional to the probability of scattering from the region outside the loss cone to the loss cone $[31,34]$.

Wall losses should be introduced into the averaged Eq. (12) according to

$$
\mathrm{v} \bar{\nu}_{\text {escape }}(\varepsilon)=\int_{\mathrm{v}_{x} \min }^{\mathrm{v}} \mathrm{v}_{x}\left[1-\exp \left(-\int_{-L / 2}^{L / 2} \frac{\nu d x}{\mathrm{v}_{x}}\right)\right] d \mathrm{v}_{x} \text {. }
$$

Equation (12) describes the change of electron density with given total energy $N(\varepsilon)=\int f(\varepsilon, x) d \mathrm{v}_{x} d \mathrm{v}_{y} d \mathrm{v}_{z} d x \delta(\varepsilon$ $\left.-m \mathrm{v}^{2} / 2+e \Phi(x)\right)$. The rate of wall loss of electrons is given by wall flux $\left.\mathbf{v}_{x} f\right|_{x= \pm L / 2}$, and the total loss of particles with given total energy is

$$
\begin{aligned}
{\left.[\partial N(\varepsilon) / \partial t]\right|_{w}=} & \int f(\varepsilon, \pm L / 2) \mathrm{v}_{x} d \mathrm{v}_{x} \\
& d \mathrm{v}_{y} d \mathrm{v}_{z} \delta\left(\varepsilon-m \mathrm{v}^{2} / 2+e \Phi_{w}\right)
\end{aligned}
$$

Integration over the perpendicular kinetic energy yields the previous result, i.e., Eq. (18). In a plasma with a nonuniform potential it is more convenient to integrate over the perpendicular energy $\left[\varepsilon_{\perp}=m\left(\mathrm{v}_{y}^{2}+\mathrm{v}_{y}^{2}\right) / 2\right]$, since it is conserved during collisionless motion:

$$
\begin{aligned}
& \overline{\mathrm{V} \nu_{\text {escape }}}=\int_{0}^{\varepsilon+e \varphi_{w}}\left[1-\exp \left(-\int_{-L / 2}^{L / 2}\right.\right. \\
& \left.\left.\times \sqrt{\left(\frac{\varepsilon+e \Phi(x)}{\varepsilon+e \Phi(x)-\varepsilon_{\perp}}\right)} N_{a} \sigma_{\text {ea }}^{\text {total }} d x\right)\right] d \varepsilon_{\perp} / m .
\end{aligned}
$$

In Fig. 7, EDF's calculated using the averaged kinetic equation and the MC method are compared. Both EDF's agree well for energies up to $\varepsilon<-2 \Phi_{w}$. For higher energies the values of the EDF calculated by MC code are much higher than theoretical predictions. The reasons are not clear. Possibly the concept of diffusion in energy fails, since electrons of these energies are not trapped and leave the discharge with a frequency approximately equal to the bounce frequency. This implies that there are no longer many bounces in the resonance region, and averaging over many interactions with resonance is not adequate. Other reasons for the discrepancy mentioned above could be the numerical limits of the MC simulation, particularly the finite period of the random number generator (RNG). This could be so in spite of the good quality of the RNG used in the simulation because of the large range of EDF values under investigation (more than four orders of magnitude). We discuss these effects elsewhere.

For an inhomogeneous magnetic field, averaging should be performed accordingly:

$$
\begin{aligned}
& \overline{\mathrm{V} \nu_{\text {escape }}}=\sum_{ \pm} \int_{0}^{\mu_{\max }}\left[1-\exp \left(-\int_{-L / 2}^{L / 2}\right.\right. \\
& \left.\left.\times \sqrt{\left(\frac{\varepsilon+e \Phi(x)}{\varepsilon+e \Phi(x)-\mu B(x)}\right)} N_{a} \sigma_{\text {ea }}^{\text {total }} d x\right)\right] \\
& \times \frac{B( \pm L / 2)}{2 m} d \mu,
\end{aligned}
$$


a)

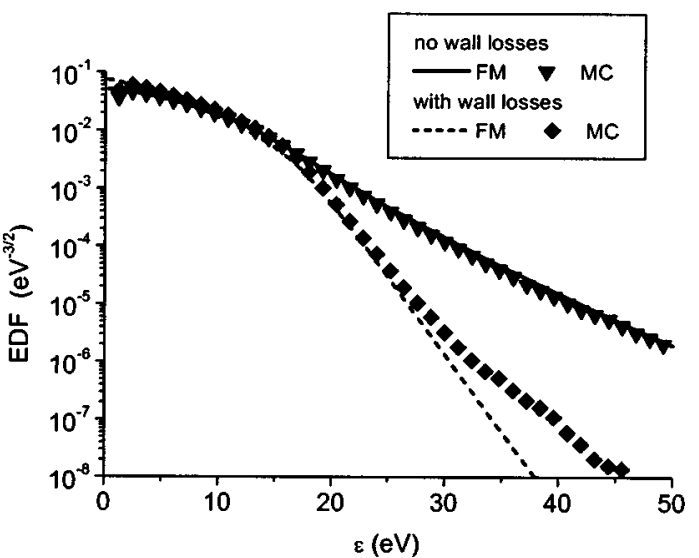

b)

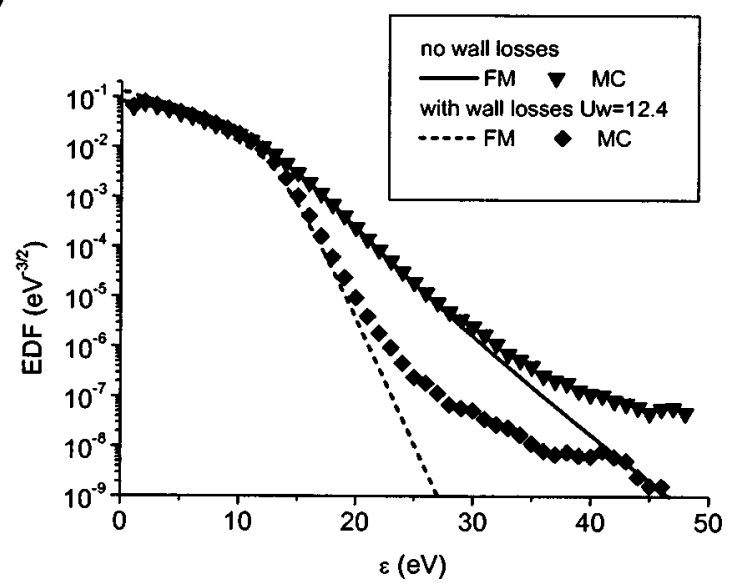

FIG. 7. The EDF's calculated from the averaged kinetic equation (lines) and by the MC method (symbols) as functions of the total energy with account for wall losses (dashed lines) and without account of wall losses (solid lines); $E_{\perp}=0.7 \mathrm{~V} / \mathrm{cm}$. (a) $p$ $=0.25 \mathrm{~m}$ Torr, $-\Phi_{w}=16.4 \mathrm{eV}$, (b) $p=1 \mathrm{~m}$ Torr, $-\Phi_{w}=14.2 \mathrm{eV}$.

where the sum is taken over the left $(-)$ and right $(+)$ loss cones, and $\mu_{\max }=m\left(\varepsilon+e \Phi_{w}\right) / B( \pm L / 2)$.

\section{SELF-CONSISTENT FAST MODELING OF ELECTRON CYCLOTRON RESONANCE DISCHARGE WITH THE USE OF ELECTRON BOLTZMANN KINETIC EQUATION AVERAGED OVER FAST ELECTRON BOUNCING AND PITCH-ANGLE SCATTERING}

In this section we summarize previous results and present a full system for the modeling of ECR discharges. Examples of calculations will be given at the end of this section.

The self-consistent set of equations is very similar to the one we used for the modeling of capacitively coupled plasmas [12]. To obtain a complete set of equations, it is necessary to determine electron density and ionization rate via the EDF:

$$
\frac{4 \pi}{m} \int_{-e \Phi(x)}^{\infty} F_{0}(\varepsilon) \sqrt{\left(\frac{2}{m}(\varepsilon+e \Phi(x))\right)} d \varepsilon=n_{e}(x),
$$

$$
\begin{aligned}
R_{\text {ion }}(x, t)= & \frac{4 \pi \sqrt{2}}{m^{3 / 2}} \int_{-e \Phi(x)+I}^{\infty}\left[N_{a} \vee \sigma_{\text {ion }}(\varepsilon+e \Phi(x))\right] \\
& \times F_{0}(\varepsilon) \sqrt{\varepsilon+e \Phi(x)} d \varepsilon
\end{aligned}
$$

where $N_{a}$ is the neutral atom density, $\sigma_{\text {ion }}$ is the ionization cross section, and $I$ is the ionization threshold.

The quasineutrality condition determines the ambipolar potential

$\frac{4 \pi}{m} \int_{-e \Phi(x)}^{\infty} F_{0}(\varepsilon) \sqrt{\left(\frac{2}{m}(\varepsilon+e \Phi(x))\right)} d \varepsilon=n_{i}(x)$,

The boundary conditions are of the form $\left.\Phi\right|_{x=0}$ $=0,\left.(d \Phi / d x)\right|_{x=0}=0$. The ion mean free path is much smaller than that of the electrons, so we assume that the ion mean free path is smaller than the discharge width. In the general case the ion kinetic equation should be solved. The ion continuity equation reads

$$
\frac{\partial n_{i}}{\partial t}+\frac{\partial n_{i} u_{i}}{\partial x}=R_{\text {ion }}(x, t),
$$

where $R_{\text {ion }}(x, t)$ is the ionization rate. For the determination of the ion velocity we use the nonlinear ion mobility taken from Ref. [12]. The boundary conditions have to be applied at the sheath boundary: $u_{i}( \pm L / 2)=u_{B} \equiv \sqrt{T_{e} / M}$, where $T_{e}$ $=-(d \ln f / d \varepsilon)_{\varepsilon=-\varphi(L / 2)}^{-1}$; we used the generalized Bohm criterion for an arbitrary EDF $[14,35]$.

The electron kinetic equation (11) taking account of losses to the walls and electron-electron collisions, reads

$$
\begin{aligned}
-\frac{d}{d \varepsilon}\left(\left[\overline{\mathrm{v} D_{\varepsilon}}(\varepsilon)+\overline{\mathrm{v} D_{\varepsilon e e}}(\varepsilon)\right] \frac{d F_{0}(\varepsilon)}{d \varepsilon}\right) & -\frac{d}{d \varepsilon}\left(\overline{\mathrm{v} V_{\varepsilon e e}}(\varepsilon) F_{0}(\varepsilon)\right) \\
= & -\left[\overline{\mathrm{v} \nu_{\text {escape }}}+\sum_{k} \overline{\mathrm{v} \nu_{k}^{*}}(\varepsilon)\right] F_{0}(\varepsilon) \\
& +\sum_{k} \overline{\mathrm{v} \nu_{k}^{*}}\left(\varepsilon+\varepsilon_{k}^{*}\right) F_{0}\left(\varepsilon+\varepsilon_{k}^{*}\right) \\
\left(\mathrm{v} V_{\varepsilon}\right)_{e e}= & \frac{m \mathrm{v}^{3}}{2 n(x)} 8 \pi \nu_{e e}(\mathrm{v}, x) \int_{e \Phi(x)}^{\varepsilon} d \varepsilon \frac{\mathrm{v}}{m} F_{0}(\varepsilon), \\
\left(\mathrm{v} D_{\varepsilon}\right)_{e e}= & \frac{4 \pi}{3} \frac{m \mathrm{v}^{3}}{n(x)} \nu_{e e}(\mathrm{v}, x)\left[\int_{-e \Phi(x)}^{\varepsilon} d \varepsilon \frac{\mathrm{v}^{3}}{m} F_{0}(\varepsilon)\right. \\
& \left.+\frac{\mathrm{v}^{3}}{m} \int_{\varepsilon}^{\infty} d \varepsilon F_{0}(\varepsilon)\right]
\end{aligned}
$$

where

$$
\begin{gathered}
\nu_{e e}(\mathrm{~V}, x)=\left[4 \pi e^{4} n(x) \ln \Lambda_{e e}\right] / m^{2} \mathrm{~V}^{3}, \\
\mathrm{~V}=\sqrt{(2 / m)(\varepsilon-e \Phi(x))},
\end{gathered}
$$

and $\ln \Lambda_{e e}$ is the Coulomb logarithm, and $k$ enumerates inelastic processes (excitation and ionization): 

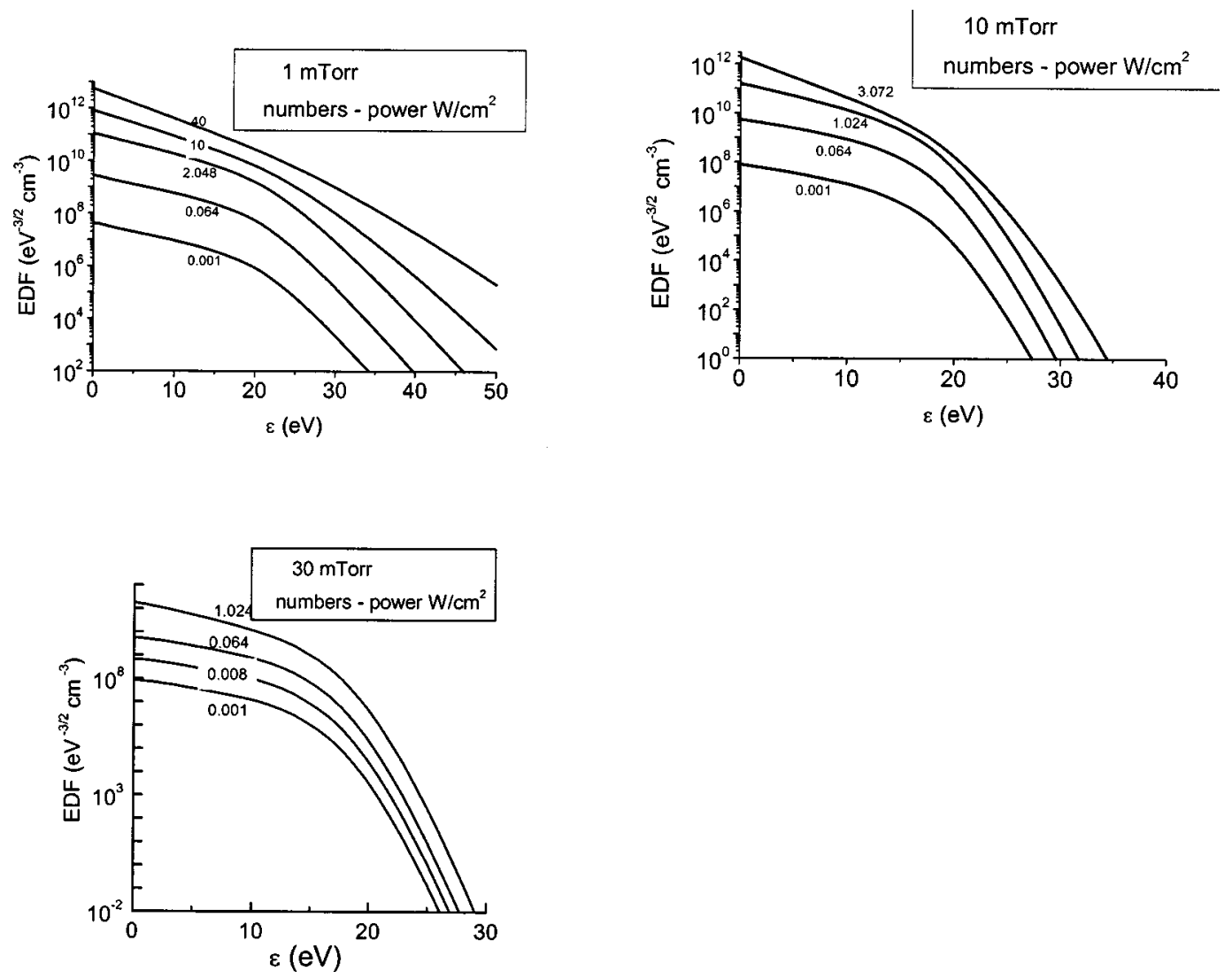

FIG. 8. The EDF's as functions of the total energy calculated from the self-consistent set of equations (24). The ECR resonance is in the discharge center.

$$
\begin{aligned}
& \overline{\mathrm{V} \nu_{\text {escape }}}=\sum_{ \pm} \int_{0}^{\mu_{\max }}\left[1-\exp \left(-\int_{-L / 2}^{L / 2}\right.\right. \\
& \left.\left.\times \sqrt{\left(\frac{\varepsilon+e \Phi(x)}{\varepsilon+e \Phi(x)-\mu B(x)}\right)} N_{a} \sigma_{\text {ea }}^{\text {total }} d x\right)\right] \\
& \times \frac{B( \pm L / 2)}{2 m} d \mu,
\end{aligned}
$$

where the sum is taken over left $(-)$ and right $(+)$ loss cones, $\mu_{\max }=m\left(\varepsilon+e \Phi_{w}\right) / B( \pm L / 2)$,

$$
\langle\overline{\mathrm{v} D}\rangle_{\varepsilon}=\frac{4 \pi}{3} \frac{\left(e E_{\perp} \varepsilon\right)^{2}}{m\left|\frac{d \omega_{B}}{d x}\right| \sqrt{2 m \varepsilon}},
$$

and the upper bar denotes only averaging in the coordinate at constant total energy:

$$
\overline{G(\mathrm{v})}=\int_{x_{-}(\varepsilon)}^{x_{+}(\varepsilon)} d x G[\sqrt{2(\varepsilon+e \Phi(x)) / m}] .
$$

The last term on the right-hand side of Eq. (24) describes the production of slow electrons in inelastic collisions. In the ionization processes two electrons are created so the ionization income term is a bit more complicated than the one for excitation. We assume that progeny electrons are born according to the probability distribution function $S(W, E)$ $=\sigma_{\text {ion }}(E) P(W, E)$, where $W$ is the energy of the progeny electrons, $E$ is the energy of the primary electron, $\sigma_{\text {ion }}$ is the ionization cross section, and $\int_{0}^{(E-I) / 2} P(W, E) d W=1$. Progeny electrons have lower energy than primary electrons, $W$ $<(E-I) / 2$, by definition. According to the Pauli principle, electrons are not distinguishable, so we cannot differentiate which electron was primary. For example, in Ref. [36] the probability function was taken in the form

$$
P(W, E)=\frac{B}{\left(W^{2}+B^{2}\right) \arctan \left[\frac{E-I}{2 B}\right]},
$$

and $B$ was set to $10 \mathrm{eV}$. For $(E-I)<2 B$ we obtain $P(W, E)=2 /(E-I)$, which is uniform in the energy of progeny electrons. For $(E-I) \gg 2 B$ we obtain $P(W, E)$ $\approx 1 / 2 \delta(W)$, i.e. all progeny electrons are created at low energies. Summing all possible income channels, the rate of production of slow electrons with energy $W$ is

$$
R_{\text {ion }}(W)=\int_{\varepsilon+1}^{\infty} P_{\Sigma}(W, E) \sqrt{2 E / m} \nu_{\text {ion }}(E) F_{0}(E) d E
$$

where $\nu_{\text {ion }}=\mathrm{v} \sigma_{\text {ion }}$ is the ionization frequency, and

$$
P_{\Sigma}(W, E)=\left\{\begin{array}{cc}
P(W, E), & W<(E-I) / 2 \\
P(E-I-W, E), & W>(E-I) / 2
\end{array}\right.
$$

is the probability for an electron with kinetic energy $E$ to create an electron with kinetic energy $E$. For example, for the 
TABLE I. The effective temperatures, wall potential, two-thirds of the mean electron energy, potential at the plasma-sheath boundary, and the potential drop in the sheath at a low power density.

\begin{tabular}{cccccccc}
\hline \hline $\begin{array}{c}p \\
\text { (m Torr) }\end{array}$ & $\begin{array}{c}\frac{2}{3}\langle\varepsilon\rangle \\
(\mathrm{eV})\end{array}$ & $\begin{array}{c}\Phi_{\mathrm{sh}}, \\
(\mathrm{eV})\end{array}$ & $\begin{array}{c}T_{\mathrm{sh}}, \\
(\mathrm{eV})\end{array}$ & $\begin{array}{c}\Phi_{w} \\
(\mathrm{eV})\end{array}$ & $\begin{array}{c}T_{w} \\
\mathrm{eV}\end{array}$ & $\begin{array}{c}\left(\Phi_{\mathrm{sh}}-\Phi_{w}\right) / 4.7 \\
(\mathrm{eV})\end{array}$ & $\begin{array}{c}F_{0}\left(\Phi_{w}\right) \\
F_{0}\left(\Phi_{\mathrm{sh}}\right)\end{array}$ \\
\hline 1 & 5.0 & 3.8 & 6.6 & 20.7 & 2.2 & 3.6 & 39 \\
10 & 4.0 & 7.5 & 4.8 & 17.4 & 1.4 & 2.1 & 50 \\
30 & 3.6 & 9.1 & 3.6 & 16.7 & 1.1 & 1.6 & 58 \\
\hline \hline
\end{tabular}

simplest case when both primary and progeny electrons are assumed to be born with the same energy $\frac{1}{2}(E-I), \quad P(W, E)=2 \delta(W-(E-I) / 2) \quad$ and $\quad R_{\text {ion }}(W)$ $=4 \sqrt{2(2 W+I) / m} \nu_{\text {ion }}(2 W+I) F_{0}(2 W+I)$.

The wall potential is to be found from the balance of the ion and electron fluxes to the wall: $\Gamma_{\mathrm{ew}}=\Gamma_{\mathrm{iw}}$ :

$$
n(L / 2) u_{B}=\frac{4 \pi}{m} \int_{-e \Phi(x)}^{\infty} F_{0}(\varepsilon) \overline{\mathrm{V} \nu_{\text {escape }}(\varepsilon)} d \varepsilon .
$$

The electric field in the resonance point can be related to the total power dissipated in the discharge. The easiest way to calculate the power density is to integrate the electron-wave interaction integral in the form of Eq. (5): integration $(1 / m) \int d \mu d \mathrm{v}_{I I}(\varepsilon-e \Phi) \int d x B(x) S_{t}^{\mathrm{ECR}}(f)$ yields the total power density deposited into the discharge. After two partial integrations,

$$
\begin{aligned}
P & =\frac{1}{m} \int d \mu d \mathrm{v}_{I I} F_{0}\left[\mathrm{v}_{I I}\left(\frac{\partial}{\partial \mu} D_{\mu}\right)\right] \\
& =n\left(x_{r}\right)\left[B \mathrm{v}_{I I}\left(\frac{\partial D_{\mu}}{\partial \mu}\right)\right],
\end{aligned}
$$

Fortunately the last factor in brackets $\mathrm{v}_{I I}\left(\partial D_{\mu} / B \partial \mu\right)$ $=\pi e c E_{\perp}^{2} /|d B / d x|$ is a constant. The power deposited into the discharge per unit area is determined only by the plasma density, the electric field amplitude, and the derivative of the magnetic field in the resonance point, and does not depend on the EDF form.

Thus the self-consistent description of the ECR discharge is completed. The full set of equations includes the electron kinetic equation (24), the ion continuity equation (23), and the ionization source term in the form of Eq. (21); the equation for the sheath potential equation (26); the power deposited in the discharge [Eq. (27)]; and the quasineutrality condition represented by Eq. (22).

In Fig. 8, the EDF evolution versus discharge power density is plotted for three pressures-1, 10, and $30 \mathrm{~m}$ Torr. One can see that at low discharge power densities the EDF's are similar. At higher powers $\left(\sim 10 \mathrm{~W} / \mathrm{cm}^{2}\right)$ the EDF's approach a Maxwellian shape due to the influence of electron collisions. At lower powers the EDF is strongly non-Maxwellian. The abrupt drop in the EDF's due to losses to the walls starts at the energy of the wall potential, $\Phi_{w}$. The corresponding slope of the EDF is characterized by an effective temperature $T_{\text {eff }}(\varepsilon) \equiv\left(d \ln \left[F_{0}(\varepsilon)\right] / d \varepsilon\right)^{-1}$ and is presented in Table I for lower powers, i.e., where all EDF's are similar. As can be seen, for lower pressure $T_{\text {eff }}\left(-e \Phi_{w}\right)$ increases, which is a common property of all discharges. The effective temperature in the tail is about three times lower than two thirds of the average kinetic energy. In the case of a Maxwellian EDF they should coincide. The large difference indicates the importance of accurate calculation of losses in the EDF tail.

In Fig. 9 the EDF's depicted for the case of ECR resonance are shifted from the center toward one of the walls at one-fourth of the gap. The electrons trapped in the potential well in the center are not heated and have a very low tem-
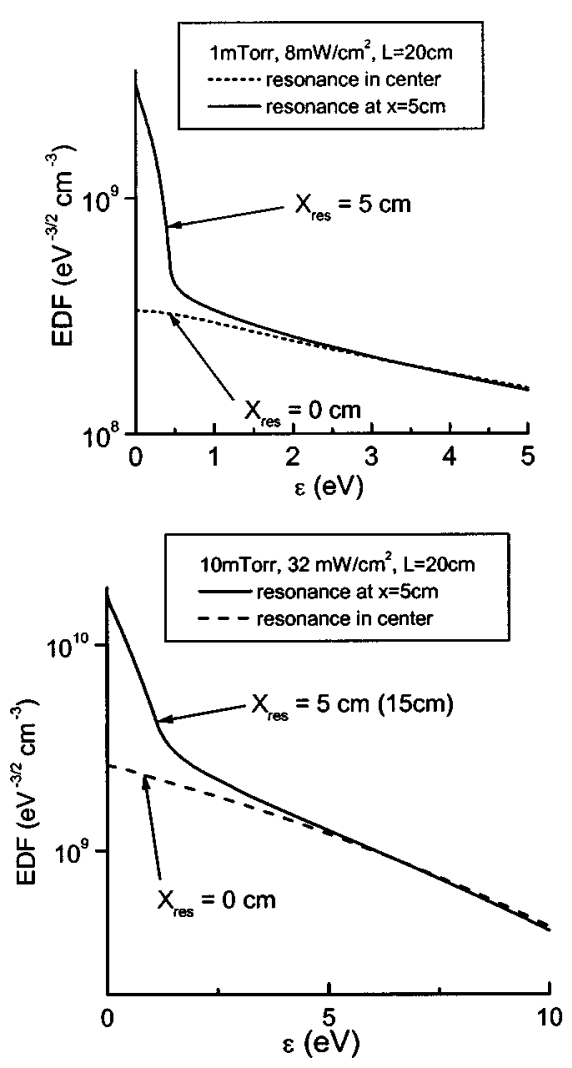
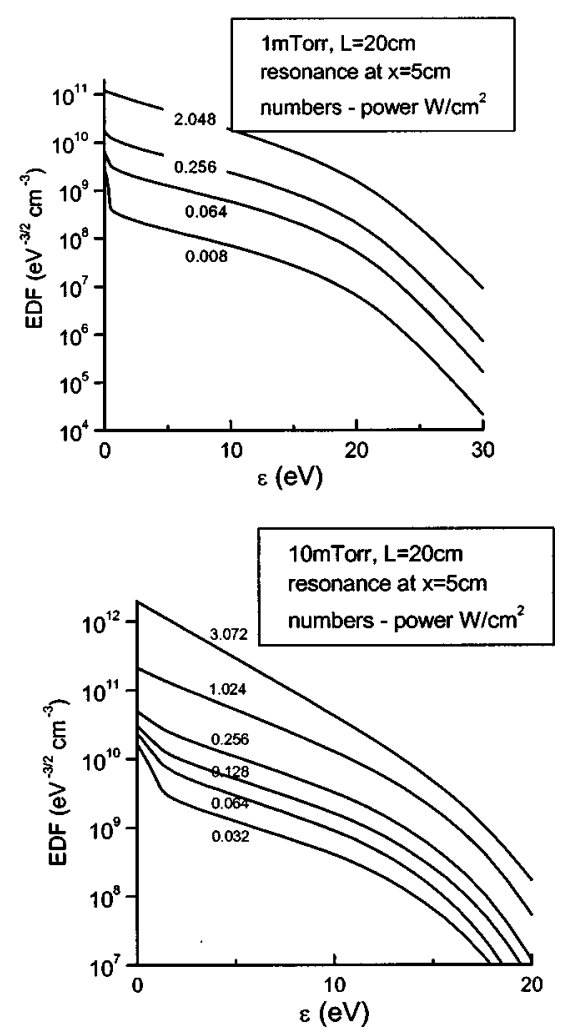

FIG. 9. The EDF's as functions of the total energy calculated from the self-consistent set of equations (24). The ECR resonance is shifted to one-fourth of the gap. 

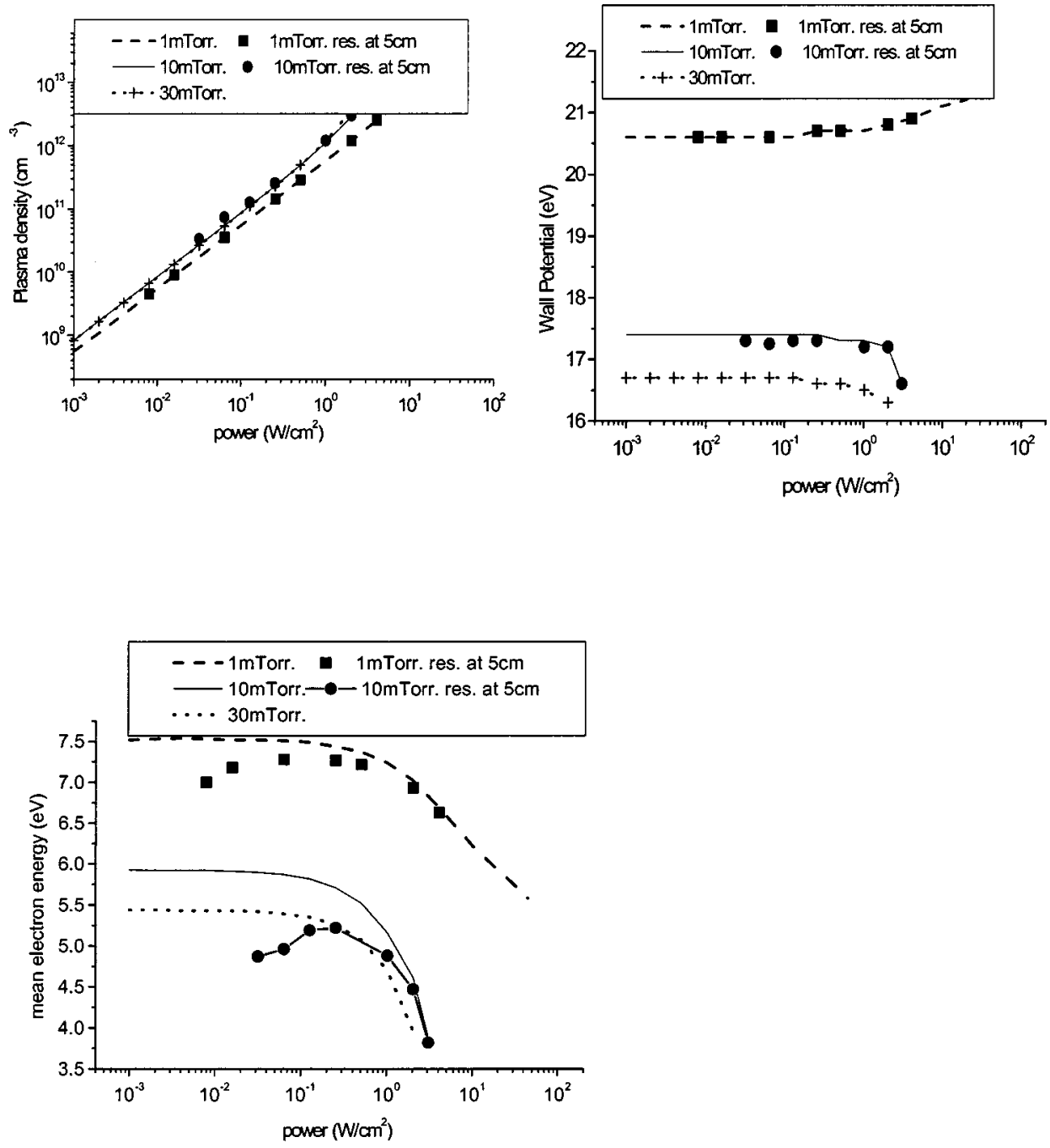

perature, determined by the energy exchange in electronelectron collisions with other electrons, energetic enough to reach the ECR resonance. For a higher plasma density the energy exchange is more effective, so the cold part of the EDF approaches the main part. In contrast, at low powers the temperature of the cold electrons can be as low as room temperature, similar to the case of the negative glow in a direct current discharge or an afterglow [37]. The presence of cold electrons was observed experimentally [38], and will be considered in Sec. IX.

The central plasma density, wall potential $\Phi_{w}$, the mean electron energy, the potential at the plasma-sheath boundary $\Phi_{\text {sh }}$, and the potential difference in the sheath $\Phi_{w}-\Phi_{\text {sh }}$ are shown as functions of the power density in Figs. 10 and 11. Since the EDF form changes only slightly at low powers, the wall potential, the potential at the plasma-sheath boundary, and the mean electron energy do not vary much with power, and the plasma density is proportional to the power density.

The sheath potential is governed by the ratio of the central and boundary densities, and decreases with pressure according to Bohm's criterion. The effective frequency of wall losses [see Eq. (24)] is typically higher than the ionization frequency at these low pressures. The total electron flux to the wall and the total number of ionizations are equal to the ion flux to the wall [Eq. (26)]. This implies that the wall potential is higher than the ionization potential and increases
FIG. 10. The plasma density, wall potential, and mean electron energy as functions of power per unit area from the self-consistent set of equations (24). with pressure, since the temperature in the EDF tail also increases.

The potential drop in the sheath-the difference in the wall and sheath potential $\Phi_{w}-\Phi_{\text {sh }}$-appears to equalize the electron flux to the wall (in the case of Maxwell EDF $\left.n(L / 2) \sqrt{T_{e} / 2 \pi m}\right)$ and the ion flux $n(L / 2) \sqrt{T_{e} / M}$. For a Maxwellian EDF it yields $\Phi_{w}-\Phi_{\mathrm{sh}}=1 / 2 e T_{e} \ln (M / 2 \pi m)$ $=4.7 T_{e} / e$ for argon. In reality the EDF is not Maxwellian and not even isotropic. For example, as can be seen from Table I, the electron temperature can change by a factor of 3 in the range $-e \Phi_{\mathrm{sh}}<\varepsilon<-e \Phi_{w}$. At not very low pressures, when the electron mean free path is smaller than the gap, the loss cone is "filled" and the EDF is close to isotropic; see Sec. VII. So we can expect $4.7 T_{e}\left(-e \Phi_{w}\right)<e\left|\Phi_{w}-\Phi_{\mathrm{sh}}\right|$ $<4.7 T_{e}\left(-e \Phi_{\mathrm{sh}}\right)$, as can be seen in Table I. In the case of a Maxwellian EDF the ratio of EDF's $F_{0}\left(-e \Phi_{\mathrm{sh}}\right) / F_{0}$ $\left(-e \Phi_{w}\right)$ is $\sqrt{M / 2 \pi m}=111$ for argon. Due to a large variation of the effective temperature this ratio is smaller by a factor of 2-3 for EDF's in Figs. 8-11; see Table I.

\section{COMPARISON OF EXPERIMENTAL DATA AND FM RESULTS}

We compared the FM results with optical measurements of the EDF's in an ECR nitrogen discharge [39] driven by microwaves at a frequency of $9.7 \mathrm{MHz}$, a pressure of 2.4 

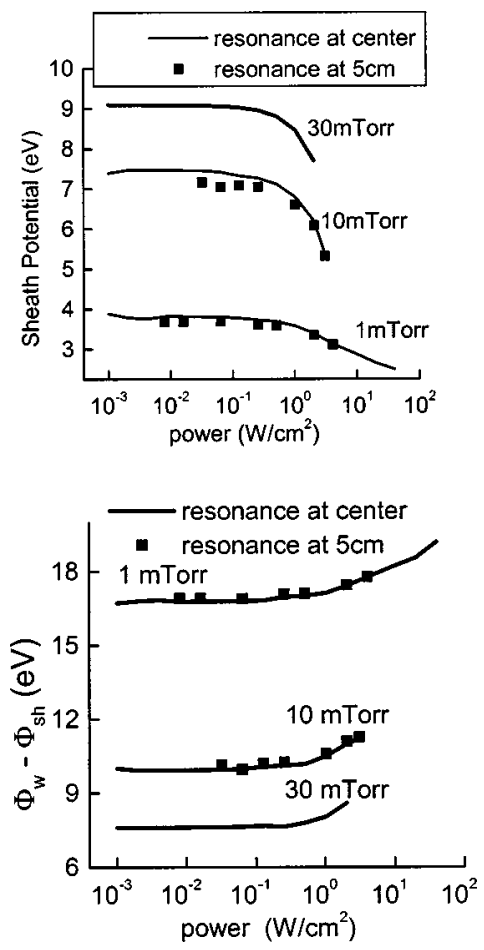

FIG. 11. The potential at the plasma-sheath boundary $\left(\Phi_{\mathrm{sh}}\right)$ and potential drop in the sheath $\left(\Phi_{w}-\Phi_{\mathrm{sh}}\right)$ as a function of power density from the self-consistent set of equations (24).

$\times 10^{-5}$ Torr, and a power of $100 \mathrm{~W}$ [2]. Unfortunately the radial distribution of the microwave power density was not reported in this experiment, so we could not perform a detailed comparison with the self-consistent modeling. That is why we assumed an average value of $0.141 \mathrm{~W} / \mathrm{cm}^{2}$ for the density per unit area, and the collisionless limit (the ion mean free path is larger than plasma gap) for the axial profile of the plasma density [14]. The plasma density of 4.0 $\times 10^{10} \mathrm{~cm}^{-3}$ was taken from the experiment. The comparison is shown in Fig. 12. As can be seen, there is a satisfactory agreement with the EDF slope in the tail, but a considerable difference in the low-energy part. The most probable reasons for this are the more complicated power deposition than what is introduced in the model, and the radial inhomogeneity of the EDF's, which are spatially averaged in the experimental data. The Doppler shift effect can be important for a proper modeling of the wave-particle interaction at high power densities [20].

To show the great influence of different processes on the EDF, we performed a number of calculations while varying power, plasma density, point of resonance, etc. Both calculations with the resonance in the center and with the resonance shifted to the periphery give the same results for the given density due to an effective energy mixing in the electron-electron collisions. We scaled down the power and plasma density in order to investigate the possibility of formation of cold electrons when the ECR resonance is shifted to one-fourth of the discharge length, i.e., out of the assumed minimum of the electron potential energy at the discharge center. As can be seen from Fig. 12, the cold electrons appear only at a considerably decreased plasma density-30 times lower than the experimental value-because at large plasma densities the electron-electron collisions effectively a)

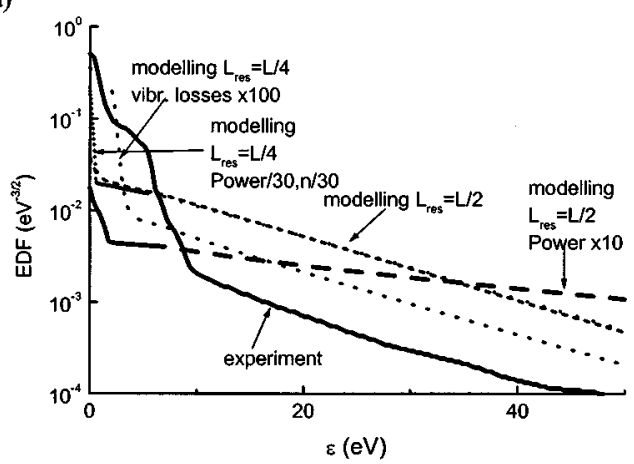

b)

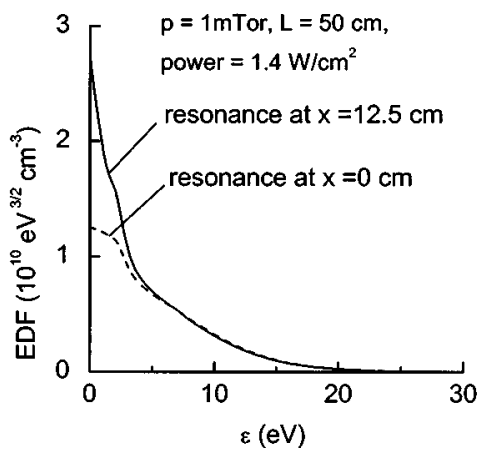

FIG. 12. The EDF's in $\mathrm{N}_{2}$ as functions of the kinetic energy (a) measured in Ref. [2] and calculated by the FM method at a power density of $0.141 \mathrm{~W} / \mathrm{cm}^{2}$ and a plasma density of $4.0 \times 10^{10} \mathrm{~cm}^{-3}$. For comparison, the number of calculated EDF's are presented for various cases: artificially increased losses due to vibrational excitation (100 times); the power scaled up ten times; power and plasma density decreased 30 times; and a resonance point positioned at $L$ $=L / 4$. (b) Calculated EDF at a pressure of $1 \mathrm{~m}$ Torr and a power per unit area of $1.4 \mathrm{~W} / \mathrm{cm}^{2}$.

interchange energy among electrons with different energies. Note that due to the energy losses in the vibrational excitation the formation of cold electrons is more favorable in nitrogen than in noble gases. We have performed selfconsistent calculations of EDF's at a pressure of $1 \mathrm{~m}$ Torr [see Fig. 12(b)], and found the formation of cold electrons in nitrogen at powers 100 times higher than in argon. The vibrational losses are not important for EDF formation at very low pressures of $10^{-5}$ Torr, and start to be important only if artificially increased 100 times. Therefore, the actual reason for the formation of a large fraction of cold electrons in experiment [38] remains unclear.

\section{CONCLUSIONS}

An electron Boltzmann kinetic equation averaged over the fast electron bouncing and pitch-angle scattering was derived in order to develop an effective and operative tool for the fast modeling (FM) of low-pressure ECR discharges. An analytical solution for the EDF in a loss cone was derived. The validity of the fast modeling method has been proved by a comparison with Monte Carlo simulations. The complete system of equations for FM is presented and ready for use in a comprehensive study of ECR discharges. The variations of plasma density and of wall and sheath potentials are ana- 
lyzed by solving a self-consistent set of equations for the EDF in the loss cone.

\section{ACKNOWLEDGMENTS}

Helpful discussions with Professor L. Tsendin, Professor A. Valkov, and Professor D. Economou are greatly appreciated. We thank Professor N. Bibinov and Professor K. Wiesemann for informing us about their experimental results prior to publication. This work was funded by the University of Antwerp, Belgium (New Research Initiative "Mathemati- cal Simulation of Pulsed, Radio-Frequency and Magnetron Discharges', ) and by Naval Research Laboratory Contract No. 68171-97-M-5379. The work of I. K. and M. M. was supported by the Belgian Office for Scientific Technical and Cultural Affairs, Scientific and Technical Collaboration with Central and East Europe. I. K. was also partially supported by the National Science Foundation, Contract No. CTS9713262, B. S. was supported, in part, by INTAS, Grant No. 96-0235, by the Russian Foundation for Basic Research, Project No. 98-02-16000, and by "UTS $i$ Plasmennye Processy"' N378.
[1] M. A. Lieberman and A. J. Lichtenberg, Principles of Plasma Discharges and Materials Processing (Wiley, New York, 1994).

[2] N. K. Bibinov, D. O. Bolshukhin, and K. Wiesemann, Rev. Sci. Instrum. 69, 1200 (1998); and private communication.

[3] V. A. Zhil'tsov, A. A. Skovoroda, and A. V. Timofeev, Fiz. Plazmy 17, 771 (1991) [Sov. J. Plasma Phys. 17, 447 (1991)].

[4] S. V. Berezhnoi, I. D. Kaganovich, and L. D. Tsendin, Fiz. Plazmy 24, 603 (1998) [Plasma Phys. Rep. 24, 556 (1998)].

[5] L. L. Beilinson, V. A. Rozhansky, and L. D. Tsendin, Phys. Rev. E 50, 3033 (1994).

[6] V. A. Godyak and R. B. Piejak, Phys. Rev. Lett. 65, 996 (1990).

[7] V. A. Godyak and R. B. Piejak, Phys. Rev. Lett. 81, 369 (1998).

[8] R. R. Araslanbekov, A. A. Kudravtsev, Phys. Rev. E 58, 7785 (1998).

[9] C. K. Birsdall, IEEE Trans. Plasma Sci. 19, 65 (1991).

[10] Glenn Joyce, Martin Lampe, and Wallace M. Manheimer, J. Comput. Phys. 138, 540 (1997).

[11] M. Lampe, G. Joyce, W. M. Manheimer, and S. P. Slinker, IEEE Trans. Plasma Sci. 26, 1592 (1999).

[12] S. V. Berezhnoi, I. D. Kaganovich, and L. D. Tsendin, Plasma Sources Sci. Technol. 7, 268 (1998).

[13] I. D. Kaganovich and L. D. Tsendin, IEEE Trans. Plasma Sci. 20, 66 (1992).

[14] I. D. Kaganovich and L. D. Tsendin, IEEE Trans. Plasma Sci. 20, 86 (1992).

[15] U. Kortshagen and L. D. Tsendin, Appl. Phys. Lett. 65, 1355 (1994).

[16] U. Kortshagen, I. Pukropski, and L. D. Tsendin, Phys. Rev. E 51, 6063 (1995).

[17] M. A. Lieberman and A. J. Lichtenberg, J. Plasma Phys. 15, 125 (1973)

[18] A. V. Timofeev and M. D. Tokman, Fiz. Plazmy 20, 377 (1994) [Plasma Phys. Rep. 20, 336 (1994)].

[19] A. V. Kasheev and N. V. Suetin, IEEE Trans. Plasma Sci. 23, 591 (1995).
[20] M. Lampe, W. M. Manheimer, G. Joice, R. F. Fernsler, and S. P. Slinker, Bull. Acad. Sci. USSR, Atmos. Oceanic Phys. 43, 1790 (1998).

[21] F. Jaeger, A. J. Lichtenberg, and M. A. Lieberman, J. Plasma Phys. 14, 1073 (1972).

[22] A. V. Timofeev, in Reviews of Plasma Physics, edited by B. B. Kadomtsev (Energoatomizdat, Moscow, 1985), Vol. 14, p. 56.

[23] A. V. Zvonkov and A. V. Timofeev, Fiz. Plazmy 23, 945 (1997) [Plasma Phys. Rep. 23, 872 (1997)].

[24] I. D. Kaganovich, Phys. Rev. Lett. 82, 327 (1999).

[25] Y. M. Aliev, I. D. Kaganovich, and H. Schlülter, Phys. Plasmas 4, 2413 (1997).

[26] A. P. Dmitriev and L. D. Tsendin, Zh. Eksp. Teor. Fiz. 81, 2032 (1981) [Sov. Phys. JETP 54, 1071 (1982)].

[27] L. D. Tsendin, Plasma Sources Sci. Technol. 4, 200 (1995).

[28] M. Surendra, D. B. Graves, and G. M. Jellum, Phys. Rev. A 41, 1112 (1990).

[29] F. J. de Heer, R. H. J. Jansen, and W. van der Kaay, J. Phys. B 12, 979 (1979).

[30] A. Bogaerts (private communication).

[31] U. Kortshagen, G. J. Parker, and J. E. Lawler, Phys. Rev. E 54, 6746 (1996).

[32] L. Sirghi, K. Ohe, and T. Kimura, Phys. Plasmas 4, 1160 (1997).

[33] V. A. Rozhanskii and L. D. Tsendin, Fiz. Plazmy 4, 388 (1978) [Sov. J. Plasma Phys. 4, 218 (1978)].

[34] L. D. Tsendin, Zh. Eksp. Teor. Fiz. 66, 1636 (1974) [Sov. Phys. JETP 39, 805 (1974)].

[35] K.-U. Riemann, J. Phys. D 23, 493 (1991).

[36] M. Surendra, D. B. Graves, and I. J. Morey, Appl. Phys. Lett. 56, 1022 (1990).

[37] R. R. Araslanbekov and A. A. Kudravtsev, Phys. Rev. E 58, 6539 (1998).

[38] N. Bibinov, D. Bolshukhin, V. Bratsev, D. Kokh, K. Wiesemann, and U. Wolters, Rev. Sci. Instrum. 69, 1200 (1998).

[39] The cross-section set was taken from ftp//jila.colorado.edu/ collision_data/electron.txt 